\title{
1 A Bacterial Signaling Network Controls Antibiotic Resistance by Regulating Anaplerosis of
}

\section{2-oxoglutarate}

5 M. N. Hurst ${ }^{1}$, C. J. Beebout ${ }^{1}$, R. Mersfelder ${ }^{2}$, A. Hollingsworth ${ }^{2}$, K. R. Guckes ${ }^{1, \#, ~ T . ~ B e r m u d e z ~}{ }^{1}$,

K. A. Floyd ${ }^{1, \dagger}$, S. A. Reasoner ${ }^{1}$, D. Williams ${ }^{2}$, and M. Hadjifrangiskou ${ }^{1,3,4, *}$

$7{ }^{1}$ Division of Molecular Pathogenesis, Department of Pathology, Microbiology \& Immunology,

8 Vanderbilt University Medical Center, Nashville, TN, USA

$9 \quad{ }^{2}$ Department of Biological Sciences, Vanderbilt University, Nashville, TN, USA

$10{ }^{3}$ Department of Urology, Vanderbilt University Medical Center, Nashville, TN, USA

$11{ }^{4}$ Vanderbilt Institute for Infection, Immunology \& Inflammation, Nashville, TN, USA

14 *Please address correspondence to Lead Contact: Maria Hadjifrangiskou, Division of Molecular 15 Pathogenesis, Department of Pathology, Microbiology \& Immunology, Vanderbilt University 16 Medical Center, Nashville, TN, USA. Tel.615-322-4851.Email: maria.hadjifrangiskou@vumc.org 17 Twitter: @BacterialTalk

18 \#Present Address: Department of Biochemistry \& Molecular Biology, Pennsylvania State 19 University, Harrisburg, PA, USA.

20 †Present Address: Department of Microbiology and Environmental Toxicology, University of 21 California - Santa Cruz, Santa Cruz, CA, USA.

\section{Short Title: QseB Controls Antibiotic Resistance in E. coli}




\section{Abstract (150 words)}

25 Antibiotic resistance has become a global threat. In addition to acquiring resistance via horizontal 26 gene transfer, bacteria can evade killing by temporarily modifying their cell envelope to prevent

27 antibiotic-bacterial interactions. A critical gap in knowledge is how bacteria balance the metabolic

28 needs of altering the cell envelope with the constant need to generate energy. Cross-regulation

29 between two signaling networks in Escherichia coli increases resistance to positively charged

30 antibiotics. We show that increased resistance is supported by metabolic re-wiring controlled by

31 the QseB transcription factor. QseB controls the increase in 2-oxoglutarate required for lipid A

32 modification, by upregulating three anaplerotic pathways that feed acetyl Co-A, succinate and

33 fumarate into the TCA cycle. Exogenous addition of 2-oxoglutarate restores antibiotic resistance in

34 the qseB deletion mutant. Antibiotic resistant clinical isolates bear mutations within QseB-mediated

35 anaplerotic pathways. These findings are significant, because they uncover a previously unknown

36 mechanism of metabolic control of antibiotic resistance. 


\section{Introduction}

50 Enterobacteriaceae are common causative agents of disease in humans. Bacteria from this class

51 account for urinary tract infections, bloodstream infections and pneumonias (Paterson 2006). To

52 treat bacterial infections, antibiotics are indicated. However, antibiotic resistance in the

53 Enterobacteriaceae is on the rise, with many strains harboring mobile genetic elements that code

54 for extended spectrum $\beta$-lactamases (ESBL), which hydrolyze broad- and extended spectrum

55 cephalosporins, monobactams, and penicillins (1). Posing an even greater challenge, many

56 Enterobacteriaceae have now gained multi-drug resistance to other first line antibiotics. For

57 example, oftentimes the genes that encode ESBLs are found on the same plasmid as genes that

58 encode resistance to aminoglycosides and sulfonamides (2), while many other strains also possess

59 the ability to resist quinolones. This creates a complex challenge to treat certain Gram-negative

60 Enterobacteriaceae in the clinic. Grim statistics accompanying those of antibiotic resistance include

61 high rates of antibiotic treatment failure, with longitudinal studies indicating that one in every ten

62 antibiotic prescriptions fails even when the clinical laboratory's antimicrobial susceptibility panel

63 predicts susceptibility to a given drug regimen (3-6). The molecular underpinnings behind such

64 treatment failures remain largely undefined. This work elucidates a previously uncharacterized

65 mechanism in Escherichia coli that fuels resistance to positively charged antibiotics.

66 To treat infections caused by multi-drug resistant Enterobacteriaceae, alternative antibiotics are

67 being used, including aminoglycosides and - to a lesser extent - polymyxins (7). For example, in

68 urinary tract infections caused by ESBL- producing uropathogenic E. coli amikacin, a synthetic

69 aminoglycoside, has shown good efficacy in treatment (8). Aminoglycosides and polymyxins are

70 polycationic in nature and make contact with the bacterial cell envelope by binding to the

71 lipopolysaccharide (LPS), phospholipids, and bacterial outer membrane proteins. This interaction

72 leads increased permeability and penetration of the aminoglycoside or polymyxin into the 
73 periplasm. A mechanism used by bacteria to repel cationic antibiotics makes the bacterial cell

74 envelope less negatively charged (9).

75 Lipopolysaccharide (LPS) comprises the outer leaflet of the outer membrane of E. coli and other

76 Gram-negative bacteria. LPS is made up of a core lipid A anchor, core oligosaccharide and - in

77 several strains - $\mathrm{O}$ antigen, all of which are synthesized at the inner leaflet of the inner membrane

78 (Figure 1A). O-antigen and lipo-oligosaccharide are flipped to the periplasmic side of the inner

79 membrane by the O-antigen flippase and the MsbA flippase respectively, where O-antigen is ligated

80 onto the lipo-oligosaccharide by an enzyme called O-antigen ligase. The resulting LPS is

81 transported to the outer membrane by the Lpt transport system (10). Lipids for new LPS are tightly

82 regulated in synthesis of lipid A and arise from phospholipid biogenesis (11). Plasmid-borne

83 determinants that confer resistance to polymyxins and other cationic polypeptides primarily code

84 for enzymes that modify the charge on LPS, thereby reducing the electrostatic attraction of cationic

85 molecules to the negatively charged bacterial cell envelope. However, most of the genes associated

86 with LPS modification are also chromosomally encoded, enabling bacteria to transiently alter cell

87 envelope charge without a priori acquisition of antibiotic resistance determinants. This ability

88 contributes to the ability of bacteria to withstand antibiotic treatment (12).

89 Altering the net charge of the envelope can be accomplished through different mechanisms, several

90 of which target lipid A (13). The net charge of the membrane can be increased by dephosphorylation

91 of lipid A, or addition of positively charged groups - such as phosphoethanolamine and amino-

92 arabinose - directly to the lipid A group during synthesis (14). Addition of other groups, such as

93 glycine, as well as lipid A acylation have also been observed to increase resistance to polymyxins

94 and other cationic antimicrobial peptides. Finally, in addition to lipid A modifications, core-

95 oligosaccharide and O-antigen components can also be modified through truncation, acylation,

96 glycosylation, or addition of phosphoryl groups, colanic-, or sialic acids (9). 
97 One fundamental question that remains unanswered is how E. coli and other LPS-containing

98 microorganisms offset the metabolic burden associated with LPS modification. In E. coli, the

99 majority of LPS modifications occur during new LPS biogenesis at the cytoplasmic leaflet of the

00 inner membrane (9) and consume several products of central metabolism. This work focuses on

01 consumption of 2-oxoglutarate by the chromosomally-encoded Arn* proteins, that add a 4-amino-

02 4-deoxy-L-arabinose group onto nascent lipid A molecules. ArnA, ArnB, and ArnC convert

03 undecaprenyl-glucuronic acid to undecaprenyl phosphate- $\alpha-4$-amino-4-deoxy-L-arabinose, while

04 ArnT catalyzes the transfer of the undecaprenyl phosphate- $\alpha$-4-amino-deoxy-L-arabinose onto a

05 nascent lipid A moiety. ArnB catalyzes a transamination reaction of undecaprenyl-4-keto-pyranose

06 to undecaprenyl 4-amino-4-deoxy-L-arabinose by utilizing oxoglutarate and producing glutamate

07 in the process (Figure 1B). How the cell fulfills the higher demand for oxoglutarate in this step

08 remains unknown.

09 In previous work we determined that the PmrAB two-component system interacts - via

10 phosphotransfer events - with QseB, another transcription factor that forms a two-component

11 system with the QseC membrane-bound receptor (Figure 1C and (12, 15, 16)). Specifically, we

12 discovered that activation of the PmrB receptor by one of its ligands - ferric iron - leads to

13 phosphorylation of both the cognate PmrA and the non-cognate QseB and that both

14 phosphorylation events are necessary for E. coli to mount resistance to polymyxin B (12). Deletion

15 of the $p m r B$ receptor abolishes the ability of $E$. coli to survive polymyxin intoxication; deletion of

16 either pmrA or qseB leads to a two- to ten-fold reduction in survival, with the double deletion mutant

$17 \Delta p m r A \Delta q s e B$ phenocopying the $p m r B$ receptor mutant (12). While PmrA regulates the expression

18 of LPS-modifying enzymes in both Salmonella and E. coli $(17,18)$, the precise role of QseB in

19 mediating resistance to polymyxin B in E. coli is unknown. Intriguingly, studies have recently

20 reported the presence of an additional qse $B C$ locus within an $m c r$-containing plasmid in a colistin- 
21 resistant isolate (19) further suggesting a role for the QseBC two-component system in LPS

22 modification.

23 In this work, we demonstrate that the QseB transcription factor mediates resistance to a range of

24 positively charged antibiotics - gentamicin, amikacin and polymyxin B - by controlling the 25 anaplerosis of oxoglutarate. QseB controls the metabolism of glutamate via three routes that feed 26 succinate, fumarate and Co-enzyme A into the tricarboxylic acid (TCA) cycle. Fueling the TCA 27 cycle at points past the succinyl-CoA step serves to mitigate the loss of oxoglutarate to LPS 28 biogenesis, allowing the TCA cycle to continue running during production of modified LPS. 29 Accordingly, deletion of $q s e B$ leads to increased glutamate levels during antibiotic intoxication, a 30 phenomenon that is abolished in the $q s e B$ complementation strain or upon exogenous addition of 31 oxoglutarate. Deletion of QseB-regulated genes involved in oxoglutarate production or glutamate 32 utilization also phenocopy the qseB deletion phenotype. Deletion of a bacterial oxoglutarate sensor 33 also attenuates antibiotic resistance. Finally, analyses of clinical isolates with stable polymyxin B 34 resistant subpopulations identified in patients with urinary tract infections sustain polymorphisms 35 in succinate and oxoglutarate metabolism enzymes. We propose a new role for QseB in balancing 36 the metabolic requirements associated with LPS modification. These findings are significant, 37 because they uncover a previously unknown mechanism of metabolic control of antibiotic 38 resistance. 


\section{H1 QseBC-PmrAB signaling is active in prevalent $E$. coli clades}

12 In uropathogenic E. coli (UPEC), resistance to polymyxin B is mediated through the concerted action of two

13 interacting two-component systems $\underline{\mathbf{P} o l y} \underline{\mathbf{m} y x i n} \underline{\mathbf{r} e s i s t a n c e}(\mathrm{Pmr}) \mathrm{AB}$ and Quorum Sensing (Qse)BC (12, 15, 16,

14 20). In Salmonella spp., PmrAB orchestrates the activation of genes involved in lipid A modification in response

t5 to elevated concentrations of ferric iron, which are sensed by the PmrB sensor histidine kinase (21, 22). Activation

t6 of PmrB via auto-phosphorylation results in phosphotransfer to the cognate response regulator PmrA, which

17 transcriptionally regulates several genes involved in $\operatorname{LPS}$ modification $(13,22,23)$. In UPEC, activation of PmrB

t8 by increased levels of ferric iron leads to auto-phosphorylation and subsequent phosphotransfer to two response

19 regulators: the cognate PmrA and the non-cognate QseB $(12,16,20)$. Deletion of either qseB or pmrA leads to a

;0 2- to 10-fold reduction in the ability of E. coli to resist killing by Polymyxin B (PMB), while deletion of both

;1 response regulators phenocopies the $\mathrm{pmrB}$ deletion which is nearly 100\% sensitive to PMB (12). The QseC sensor

;2 histidine kinase - the cognate partner of QseB - is critical for system de-activation (20).

;3 We first asked whether QseBC is involved in mediating polymyxin B resistance in diverse E. coli strains. There

;4 are five prevalent phylogenetic clades that host the majority of sequenced E. coli isolates, A, E, B2, D, B1. Using

;5 a representative panel of E. coli strains that lack plasmid-borne polymyxin B resistance determinants and are

;6 sensitive to polymyxin, based on clinical testing (Figure 1D), we asked if pre-treatment with the PmrB ligand

;7 ferric iron would induce activation of the $q s e B C$ promoter, which is under the control of QseB (20, 24-27).

;8 Transcriptional profiling by reverse transcription-followed by quantitative qPCR revealed a robust activation

;9 surge (28) of the qseBC promoter in all strains tested from clades A, E, B2, D and B1 (Figure S1). Evaluation of

j0 strain survival in 2.5X the Minimum Inhibitory Concentration (MIC) of polymyxin B revealed that all tested

¡1 strains exhibited $77-100 \%$ survival compared to untreated controls which exhibited $4-22 \%$ survival. (Figure

i2 1D). Deletion of $q s e B$ or $q s e B C$ in well-characterized enterohemorragic strains $86-24$ and $87-14$ led to a

¡3 significant reduction in polymyxin B resistance compared to the wild-type parent (Figure S2), which was rescued

i4 upon extra-chromosomal complementation with a wild-type copy of qseB. Notably, strain Sakai harbors a

5 truncated, non-functional copy of QseC, a phenomenon which would suggest that in this strain background, 
56 PmrB-QseB interactions are unrestrained as previously seen for a uropathogenic E. coli strain deleted for qseC

57 (15). In UPEC strains, unrestrained interaction of PmrB and QseB leads to increased resistance to polymyxin B

i8 in a signal-independent manner (12). Indeed, strain Sakai exhibits intrinsic resistance to polymyxin B (Figure

59 S2), consistent with the model that absence of functional QseC leads to uncontrolled PmrB-to-QseB

70 phosphotransfer and subsequent intrinsic resistance to polymyxins. Supporting this notion, deletion of $q s e B$ or

71 the entire $q s e B C$ locus in this strain phenocopies the qseB deletion in the other EHEC isolates (Figure S2).

12 Combined these results indicate that transient resistance to polymyxin B in diverse E. coli clades is mediated at

73 least in part by PmrB - QseB interactions. To further probe the the mechanism by which QseB mediates antibiotic

74 resistance we used the prototypical pathogenic strain UTI89.

\section{QseB mediates resistance to positively charged antibiotics}

77 If QseB plays a critical role in supporting modification of the cell envelope to resist polymyxin B intoxication, 78 one would expect that QseB activation would lead to resistance to a broad range of positively charged antibiotics.

19 To test this hypothesis, isogenic strains lacking QseB, or carrying QseB in the native locus or extra30 chromosomally, were tested for their ability to resist gentamicin or amikacin, two aminoglycoside antibiotics that 31 are positively charged. Nitrofurantoin, which is neutral, along with polymyxin B were used as controls. Strains 32 were tested for their ability to survive a concentration of antibiotic at up to 5 times the established minimum 33 inhibitory concentration - in conditions without or with preconditioning with ferric iron. These studies indicated 34 that the strains harboring QseB (wild-type strain (Figure 2A, 2D, 2G) and the $\Delta q s e B /$ pQseB complemented 35 strain (Figure 2C, F, I)) exhibited 85-95\% survival in the presence of charged antibiotics when pre-conditioned 36 with ferric iron (purple lines, Figure 2). However, the strain lacking qseB ( $\triangle q s e B$, Figure $2 \mathbf{B}, \mathbf{E}, \mathbf{H})$ exhibited a 37 marked decline in survival regardless of the presence of ferric iron. Similarly, the uncharged antibiotic 38 nitrofurantoin led to effective bacterial killing of all genetic backgrounds and bacterial survival was not affected 
39 by the presence of QseB (Figure 2J, K, L). Combined, these data demonstrate that the QseB transcription factor

mediates resistance to positively charged antibiotics.

)1 Temporal Tracking of Gene Activation Under PmrB-Activating Conditions Reveals an Anaplerotic Circuit

\section{)2 Under QseB Control}

33 Previous studies in Salmonella and E. coli elucidated PmrA targets that are responsible for modification of the )4 LPS $(13,22,23,29)$. Previous studies in E. coli revealed that deletion of qseC confers a significant shift in the expression of genes involved in central metabolism, including - among others - genes involved in glutamate metabolism and the tricarboxylic acid cycle (26). To decipher the regulon of QseB under conditions of antibiotic stress, steady-state transcript abundance across the activation surge were tracked over time via RNA sequencing (RNAseq). In parallel, promoters bound by QseB were identified using Myc-His-tagged QseB and crosslinking,

followed by immunoprecipitation and analysis of bound DNA sequences on microarray chips (chIP-on-chip).

)0 For the RNAseq experiments, the wild-type strain UTI89 and isogenic mutants lacking qseB (UTI89 4 seB, or )1 $\quad p m r A$ and $q s e B$ (UTI89 $\Delta p m r A \Delta q s e B)$ were grown under PmrB-activating conditions $\left(100 \mu \mathrm{M}\right.$ Ferric iron, $\left.\mathrm{Fe}^{3+}\right)$ )2 and samples were obtained for RNA sequencing immediately prior to $(\mathrm{T}=0)$, as well as $15(\mathrm{~T}=15)$ and $60(\mathrm{~T}=60)$ )3 minutes post addition of ferric iron to the growth medium (Figure 3A). Output RNA sequencing data from three )4 biological repeats per strain per timepoint were analyzed using Rockhopper software (30, 31). Differential gene expression matrices within each strain were calculated to compare $\mathrm{T}=0$ to $\mathrm{T}=60$ and $\mathrm{T}=15$ minutes (Figure 3B-

C and Supplementary File 1). An additional comparison of $\mathrm{T}=15$ and $\mathrm{T}=60$ was also made (Supplementary

File 1). Transcripts with a q value lower than 0.05 were considered significant (Supplementary File 1). The RNAseq profile of the wild-type strain, UTI89, revealed a total of 829 transcripts with significantly altered abundance across time, in response to the addition of ferric iron (Supplementary File 1, worksheet 1). Of these, 49 belonged to small non-coding RNAs, while 148 were hypothetical proteins (Supplementary File 1, worksheet 1; highlighted in light- and dark gray respectively). Of the remaining 632 transcripts, 81 belonged to 12 tRNAs $(\mathrm{n}=50)$ and transcripts coding for tRNA modification- or translation-associated proteins ( $\mathrm{n}=31)$ 
13 (Supplementary File 1, worksheet 1; highlighted in light pink). The non-coding RNA transcripts, as well as

14 hypothetical and tRNA-/translation associated proteins were excluded from the heatmaps, along with 6 plasmid-

I5 associated transcripts unique to UTI89. The remaining 545 transcripts are depicted in heatmaps, according to the

16 pathways they belong to (Figure 3B-C, Supplementary Figure S3).

17 Although LPS modification genes surged over time, following stimulation with ferric iron (Figure 3B-C), the

8 most highly upregulated genes in response to ferric iron stimulation were genes that belonged to glutamate

19 metabolism and the TCA cycle (Figure 3C). The same clusters had no significant surge in the mutants lacking

20 QseB (Figure 3C-D, Supplementary File 1). These data indicate that QseB and PmrA are functionally redundant

21 in mediating transcription of LPS modification genes in pathogenic E. coli. Furthermore, these data indicate a

22 role for QseB in mediating glutamate metabolism.

23 ArnA/B and enzymes involved in glutamate metabolism, pantothenate and coenzyme A synthesis are under

24 the control of QseB.

25 To determine promoters bound by QseB, the UTI89 4 qseB strain that harbors a construct expressing Myc-His-

26 tagged QseB under an arabinose-inducible promoter $(12,15,27)$ was subjected to chIP-on-chip analyses, using

27 UTI89-specific Affymetrix chips (26). An isogenic strain harboring the pBAD-MycHis A vector was used as a

28 negative control. Cultures were grown in laboratory media in the presence of $0.02 \mu \mathrm{M}$ arabinose to ensure constant

29 expression of QseB, at concentrations similar to those we previously published as sufficient for QseBC

30 complementation (15). Pull-downs using an anti-Myc antibody were performed on six separate reactions, three

31 for the experimental and three for the control strain. Analyses of the pull-down DNA revealed a total of 169

32 unique promoters bound by QseB and absent in the negative control (Figure S4 and Supplementary File 1).

33 Sixty-three out of the 169 promoters were also reported in the microarray analyses of the qseC mutant in previous

34 studies (26). Among the promoters identified was the promoter of qseBC - consistent with QseB's ability to

35 regulate its own transcription $(15,24,26)$. Another set of targets included the promoters of $\operatorname{arn} B(y f b E), \arg F$,

$36 a s n B$, the $i l v$ cluster, $p a n B C D$ and $s u c D$ and $a s n B$ (Figure S4), as well as $g \ln K, g \ln S$ and $\operatorname{asp} S$ that could indirectly 
37 be responsible for the transcriptional effects observed in the RNAseq analyses. (Figure 3C and Supplementary

38 File 1). The genes directed by these identified promoters are involved in nitrogen assimilation, glutamate

39 metabolism, panthothenate and coenzyme A synthesis. As mentioned above, the $\operatorname{arn} B$ gene, encodes the enzyme

t0 that catalyzes transamination of undecaprenyl-4-keto-pyranose to undecaprenyl 4-amino-4-deoxy-L-arabinose

H1 (Figure 1B). This ArnB-mediated reaction consumes oxoglutarate and produces glutamate. Intriguingly, the

t2 QseB-bound promoters identified in the chIP-on-chip analyses (Figure S4, Supplementary File 1), along with

H3 the RNAseq data (Figure 3C) are connected through their involvement in glutamate metabolism (Figure 4).

14 Glutamate, aspartate and Co-enzyme A concentrations change in the absence of QseB

15 To determine whether QseB plays a role in glutamate-oxoglutarate homeostasis during the ArnB-mediated LPS

t6 modification step, the levels of metabolites along the above identified pathway (Figure 4) were measured.

17 Oxoglutarate is difficult to quantify, due to rapid degradation (32), thus oxoglutarate measurements were not

18 taken. For metabolomics measurements, samples were obtained at the same time intervals shown for the RNA

19 sequencing analyses (Figure 3A). Glutamate (Figure 5A-C), aspartate (Figure 5D-F), coenzyme A (Figure 5G-

;0 I) measurements were taken in the wild-type parent and the isogenic $\Delta q s e B$ and complemented strains, in the

¡1 presence of ferric iron alone (Figure 5, red lines) polymyxin B (PMB) alone (Figure 5, blue lines), ferric iron +

;2 PMB (Figure 5, pink lines) or no additives (Figure 5, black lines). In each panel, concentrations were normalized

;3 to the unstimulated/unchallenged control (black lines). In the wild-type background, addition of polymyxin B and

;4 ferric iron resulted in a decrease in glutamate levels compared to cells exposed to ferric iron alone or no additives

(Figure 5A). Conversely, in a $q s e B$ deletion mutant, glutamate levels remain relatively unchanged following

;6 addition of polymyxin B and ferric iron compared to ferric iron alone or with no additives (Figure 5B). Glutamate

57 levels decrease again shortly after addition of ferric iron and polymyxin $\mathrm{B}$ in a qseB deletion mutant

i8 complemented with a wild-type copy of $q s e B(\triangle q s e B / p Q s e B$, Figure 5C). These data indicate that glutamate

;9 levels change in response to polymyxin B addition in a manner that depends upon QseB. This could suggest that

50 rapid glutamate utilization occurs, following excess production during the conversion of undecaprenyl-4-keto-

$\lceil 1$ pyranose to undecaprenyl 4-amino-4-dexoy-L-arabinose. 
;2 Because glutamate can be converted to aspartate via the action of AspC (Figure 5), we also measured aspartate

i3 levels in the same samples (Figure 5D-F). Similar to glutamate, aspartate levels dropped in wild-type samples

34 treated with polymyxin B and ferric iron compared to a sample with no additives (Figure 5D). In a qseB deletion

i5 mutant aspartate levels rise after addition of polymyxin B and ferric iron compared to a sample with no additives.

j6 In a $q s e B$ deletion complemented with qseB aspartate levels remain relatively unchanged compared to a sample

77 with no additives (Figure 5F). Aspartate feeds into coenzyme A production through the pantothenate synthesis

i8 pathway (Figure 4), of which several gene products involved are direct targets of QseB (Figure 4). Following

59 addition of polymyxin B and ferric iron, coenzyme levels rapidly rise (Figure 5G) in a wild-type sample,

70 compared to a sample with no additives. However, in the qseB deletion mutant coenzyme A levels remain

71 relatively unchanged or rise slightly (Figure $\mathbf{5 H}$ ). Interestingly in this case, complementation with pQseB did not

12 complement the phenotype (Figure 5I).

Oxoglutarate rescues the antibiotic susceptibility of the qseB deletion mutant

75 The combined metabolomics, along with the RNAseq data suggest that QseB controls metabolic reactions that

76 shunt the glutamate produced from the modification of LPS towards production of co-enzyme A, which could

77 then re-enter the TCA (Figure 4), replenishing oxoglutarate levels. Moreover, if the RNAseq/chIP-on-chip

18 observations truly map a metabolic regulon under QseB control in response to LPS modification, it appears that

19 pantothenate production is also part of this process (Figure 4). To determine whether indeed these suggested

30 anaplerotic pathways are critical in mounting a response to positively charged antibiotics, two mutants were

31 created with deletions in panD and icdA respectively (Figure 4). PanD codes for an aspartate decarboxylase that

32 converts aspartate into beta-alanine, which then feeds into the pantothenate pathway eventually resulting in

33 coenzyme A production (Figure 4). The pan gene operon is under the direct control of QseB (Figure S4 and

34 Supplementary File 1). We reasoned that if the identified QseB regulon is active during LPS modification, then

35 deletion of panD, which is centrally placed in the identified pathway (Figure 4) should impair resistance to

36 polymyxin B. To determine whether the pathway feeds into the TCA cycle, we also created an icdA deletion 
37 mutant, disrupting the conversion of isocitrate to oxoglutarate (Figure 4), limiting oxoglutarate amounts for LPS

38 modification. Obtained mutants were tested in polymyxin B survival assays alongside the wild-type parental strain

39 and the isogenic $q s e B$ deletion mutant, as well as the $q s e B$ deletion mutant complemented with $q s e B$. Strains

30 were tested for their ability to survive a concentration of PMB at five times the established minimum inhibitory

)1 concentration - as determined in Figure 2A-C - in conditions without or with preconditioning with ferric iron.

22 While the wild-type and the $\triangle q s e B / \mathrm{pQseB}$ complemented strains exhibited $85-95 \%$ survival in $5 \mathrm{X}$ the PMB MIC

3 when pre-conditioned with ferric iron (Figure 6A), the $q s e B$, panD and $i c d A$ deletion mutants reproducibly

74 exhibited a $50 \%$ reduction in survival.

66 If QseB controls the oxoglutarate "budget", then susceptibility to charged antibiotics in the qseB deletion strain 77 may be due to limitation in the production of oxoglutarate. To test this hypothesis, we asked whether the 78 susceptibility of a qseB deletion mutant to positively charged antibiotics could be rescued via the addition of 99 exogenous oxoglutarate. Remarkably, addition of oxoglutarate to ferric iron/polymyxin B-treated samples of )0 $\triangle q s e B$ restored survival in $5 \mathrm{X}$ the antibiotic MIC (Figure 6B). These data indicate that oxoglutarate anaplerosis )1 controlled by QseB is necessary for mounting resistance to positively charged antibiotics.

22 Previous studies identified a two-component system, KguRS, prevalent in uropathogenic E. coli isolates that 3 senses oxoglutarate (33). Using this system as an orthologous tool to validate the requirement for oxoglutarate, )4 we asked whether a mutant lacking $k g u R S$ would have defects in modulating antibiotic resistance. We created a )5 mutant lacking kguRS in wild-type UPEC and tested its susceptibility to PMB. The resulting mutant )6 UTI89 $\triangle \mathrm{kguRS}$ demonstrated remarkable susceptibility to PMB (Figure 6C), a phenomenon that was rescued by 7 exogenous addition of oxoglutarate (Figure 6C).

)8 Together, these results indicate that oxoglutarate anaplerosis, as controlled by the QseB transcription factor is 99 critical for transient resistance to positively charged antibiotics. Furthermore, these observations uncover an 
10 intrinsic mechanism by which E. coli can survive an antibiotic treatment regimen, if a subpopulation of bacteria

11 has the PmrB-QseB-PmrA network activated.

\section{Clinical isolates with polymyxin hetero-resistance harbor mutations in QseB-regulated anaplerotic}

14 pathways.

I5 In clinical practice, up to $10 \%$ of prescribed regimens fail $(4-6,34)$, despite antibiogram outputs from the clinical

I6 laboratory that indicate bacterial susceptibility to the prescribed antibiotic regimen. Our observations suggest an

17 intrinsic mechanism that may contribute - at least in part - to treatment failure in the case of pathogenic E. coli

18 infections. To begin to address this hypothesis, we first evaluated the stochastic expression of the $q s e B$ locus in a

19 bacterial community, without the presence of the ferric iron stimulus. Utilizing a previously created promoter

20 construct fused to GFP, we assessed the activity of the promoter within bacterial biofilm communities, which are

21 formed at different niches during infection (35). Microscopy revealed the presence of cells in which Pqse::GFP

$: 2$ was active (Figure 7A), indicative of the presence of subpopulations within a bacterial community that have the

23 potential to resist treatment with positively charged antibiotics.

:4 We then asked whether we can identify clinical isolates re-wired to harbor subpopulations with induced qseB

.5 expression, or increased resistance to positively charged antibiotics. A total of 400 clinical urinary isolates from

26 the Vanderbilt University Clinical Microbiology were tested using the standard broth microdilution method

:7 (Figure 7B) and an E-test strip method in which a gradient concentration of antibiotic is secreted into the agar

28 medium (Figure 7C). Of these isolates, 4 exhibited hetero-resistance to PMB; the isolate consisted of a majority

29 population with an MIC less than $1.0 \mathrm{mg} / \mathrm{L}$, and a minority population with significant resistance to PMB (Figure

30 7B). Isolation and subsequent sequencing of the resistant subpopulations from 2 out of the 4 isolates determined

31 mutations in $\operatorname{suc} A B$, glutamate racemase ( $m u r I)$ and $\operatorname{gadX}$ (Figure 7B), which codes for the regulator of the gadAB

32 genes (Figure 4). In parallel studies, in vitro evolution of the susceptible subpopulation from each isolate in $0.75 \mathrm{X}$

33 the PMB MIC, led to increased PMB resistance by passage 2 (Figure 7C-D). Subsequent sequencing of passage 
342 - and passage 5 "resistant daughters" revealed the acquisition of mutations in the promoter region of gadX for

35 both VUTI163 and VUTI247 (Figure 7E and Supplementary File 2). Transcript evaluation of these strains also

36 indicated elevated expression of the $q s e B$ locus (Figure 7G-H). Together, these observations indicate that

37 permanent rewiring in QseB-regulated anaplerotic pathways are identified in clinical isolates and may contribute

38 to treatment failure in the clinic.

\section{Discussion}

H1 Antibiotic resistance among bacterial pathogens has become a global health threat, fueling the efforts to develop

12 new antimicrobial treatments. Bacteria can mount resistance to antibiotics through acquisition of mobile genetic

H3 elements, namely through acquisition of plasmids encoding antibiotic resistance cassettes. In many pathogens,

t4 resistance to antimicrobial agents is encoded chromosomally. In Salmonella sp. and Escherichia coli resistance

15 to a last resort antibiotic, polymyxin B, is intrinsically encoded. In these systems, resistance to polymyxin B is

t6 mediated through two-component signal transduction systems that lead to the upregulation of lipid A modifiers.

17 This process, while increasing the net charge of bacterial membrane and repelling cationic moieties, comes at a

18 metabolic cost associated with diverting central metabolites to synthesize modified LPS.

19 Here we demonstrate a metabolic circuit under the control of the QseB transcription factor that controls

;0 oxoglutarate homeostasis during the cellular response to positively charged antibiotics. Specifically, QseB

;1 controls the metabolism of by-products following amino-arabinose addition to nascent phospholipids by Arn*

;2 proteins which are activated by PmrA. ArnB is a direct target of both QseB and PmrA and catalyzes the conversion

;3 of undecaprnyl-4-keto pyranose to undecaprenyl 4-amin-4-deoxy-L-arabinose, a key step in the addition of an

;4 amino-arabinose moiety addition to a phospholipid. In this key catalytic step oxoglutarate is utilized, producing

;5 glutamate in the process. Glutamate is a critical commodity in the cell, being connected to glutamine and nitrogen

;6 metabolism (36-40). It is thus not surprising that the abundance of glutamate is under multi-level control, one that

7 is monitored - as we now show - by QseB. 
i8 QseB directly targets and controls several genes involved in the anaplerosis of oxoglutarate. The importance

;9 oxoglutarate in sustaining modified LPS biogenesis is emphasized by the observations that exogenous

j0 oxoglutarate rescues the $q s e B$ deletion mutant during antibiotic treatment. This not only underscores the need for

¡1 oxoglutarate during LPS modification, but also implicates the need for QseB's metabolic control of oxoglutarate

52 production. Metabolomic analyses tracking the abundance of glutamate, aspartate and Co-enzyme A (Figure 5)

53 - combined with the transcriptional profiling results (Figure 3), point towards the use of glutamate through the

34 pantothenate pathway and co-enzyme A production, which can then enter the TCA cycle either as acetyl-CoA or

55 succinyl-CoA (Figure 4). Likewise, conversion of glutamate to fumarate via the arg gene products would supply

j6 fumarate. Intriguingly, our data point towards the conversion of glutamate to GABA through the function of the

$57 \mathrm{gab} / \mathrm{gad}$ gene products, for eventual conversion into succinate and re-introduction into the TCA cycle. This step

is would replenish succinate, bypassing the need to convert oxoglutarate to succinate via the $s u c A B$ - and $s u c D C$ -

59 encoded complexes (Figure 4). Typically, GABA produced via the function of GadA/GadB and is exported from

70 the cell via the action of the GadC exporter. The gene organization of gadA and gadBC is such that gadBC are

71 transcribed from the same promoter, but transcripts are further processed at the gadBC intergenic region to release

12 $\operatorname{gadB}$ and $\operatorname{gad} C$ transcripts (41). In our transcriptional analyses, gadA and gadB were among the most highly

73 induced genes, but gadC transcript was not significantly altered (Figure 3 and supplementary file 1). This

74 indicates the presence of an additional mechanism that prevents GABA export so that it could potentially be used

75 to produce succinate (Figure 4). The discovery of clinical isolates that have resistant subpopulations with

76 mutations in gadX, along with the in vitro evolution results lends further support that this pathway can be exploited

77 to confer resistance to polymyxin. Notably, none of the source patients were treated with polymyxins prior to

78 isolation of the strains, as polymyxins are a last-resort antibiotic not deployed to treat UTI in the US. The presence

79 of resistant subpopulations with the ability to resist polymyxins, combined with the in vitro evolution experiments,

30 suggests that different envelope stresses may lead to mutations that alter the LPS, thereby collaterally producing

31 antibiotic-resistant bacteria. Resistance to polymyxin can be obtained quickly, and also exploits the QseB-

32 mediated metabolic pathway. We are in the process of quantifying GABA, succinate and fumarate in response to 
33 antibiotic stress in wild-type pathogenic E. coli and isogenic qseB-deletion and complemented strains and will be

34 investigating the fitness costs and benefits of the resistant subpopulations during infection.

35 We found that QseBC-PmrAB signaling is prevalent across different strains of tested pathogenic E. coli including

36 those that inhabit both intestinal and extraintestinal niches. This suggests that the mechanisms studied in this

37 paper may be applicable across the major clades of E. coli. Reports of plasmids $m c r-1$ thru $m c r-8$ report low

38 levels of polymyxin B and colistin resistance $(42,43)$. These elements lack $q s e B C$. However, a new plasmid, $m c r-$

399 , encoding both a $m c r-1$ homologue and qseBC like elements suggests the need for metabolic control during LPS

)0 modification (19). In studying emerging antibiotic resistance mechanisms, we tend to generally focus on

)1 understanding plasmid-encoding systems. Here, understanding a chromosomally encoded system may translate

22 to emerging plasmid encoded systems, and pose a threat in the clinic. Currently there are two polymyxins used

33 clinically: polymyxin B and E (also known as colistin). Though highly effective, polymyxins fell out of favor in

34 clinical practice due to concerns of renal and neurotoxicity in 1970s (44). Through the 1980s and 1990s,

75 polymyxin use was largely restricted to cystic fibrosis populations $(45,46)$. However, the use of polymyxins in

6 the clinic was reimplemented with the rise of multi-drug resistant infections in the early 2000s, where its use

77 continues $(47,48)$. Likewise, aminoglycoside antibiotics such as gentamicin and amikacin are used to treat serious

78 infections caused by facultative anaerobes (49). The finding that E. coli and potentially other Enterobacteriaceae

19 have the potential to mount a resistance response - at the subpopulation level - to these critical antibiotics raises

)0 the alarm for the need to better understand mechanisms that lead to heterogeneous induction of systems like

)1 QseBC in the bacterial pathogens. Lastly, this work demonstrates the need to understand how metabolic pathways

22 can be exploited in pathogenic bacteria and may give new insights to potential therapeutic targets.

)4 Materials and Methods

)5 Bacterial Strains, Plasmids, and Growth Conditions

)6 Bacterial strains, plasmids and primers used in this study are listed in the Table 2. Overnight growth was always ) 7 performed in liquid culture in Lysogeny Broth (Fisher) at $37^{\circ} \mathrm{C}$ with shaking, with appropriate antibiotics where 
)8 necessary. Details pertaining to growth conditions for each assay used in the study can be found in the relevant

)9 sections below.

11 Transcriptional Surge Experiments - To assess induction of qseBC, bacteria were grown in N-minimal media

$12(27,28)$ at $37^{\circ} \mathrm{C}$ with shaking $(220$ rotations per minute). $\mathrm{N}$-minimal media were inoculated with strains of interest

13 at starting optical density at a wavelength of $600 \mathrm{~nm}\left(\mathrm{OD}_{600}\right)$ of 0.05 . Strains were allowed to reach mid-

4 logarithmic growth phase $\left(\mathrm{OD}_{600}=0.5\right)$. At this time, 4-milliliters of culture was withdrawn for processing (see

15 below) and the remainder of the culture was split into two. To one of the two split cultures, ferric chloride (Fisher)

I6 was added at a final concentration of $100 \mu \mathrm{M}$, while the other culture served as the unstimulated control. Cultures

17 were returned to $37^{\circ} \mathrm{C}$ with shaking. Four-milliliter samples were withdrawn from each culture at 15- and 60-

18 minutes post stimulation for RNA processing as described below. All samples were centrifuged at $4000 \mathrm{x} \mathrm{g}$ for

1910 minutes upon collection. The supernatant was decanted and the fraction containing the cell pellet was flash

20 frozen in dry ice - ethanol and stored at $-80^{\circ} \mathrm{C}$ until RNA extraction.

\section{RNA Isolation}

23 RNA from cell pellets was extracted using the RNeasy kit (Sigma Aldrich) and quantified using Agilent 24 Technology (Agilent). A total of 3 micrograms ( $\mu \mathrm{g})$ of RNA was DNAse treated using the DNAfree kit (Ambion) 25 as we previously described $(16,20,26)$. A total of $1 \mu \mathrm{g}$ of DNAse-treated RNA was subjected to reversetranscription using SuperScript III Reverse Transcriptase (Invitrogen/ThermoFisher Scientific) and following the manufacturer's protocol.

\section{RNA Sequencing and Analysis}

30 Strains were grown in N-Minimal media at $37{ }^{\circ} \mathrm{C}$ with shaking, and samples were obtained as described for the 31 transcriptional surge experiments. RNA was extracted and DNAse-treated as described in the RNA isolation 32 section. DNA-free RNA quality and abundance were analyzed using a Qubit fluorimeter and Agilent Bioanalyzer. 33 RNA with an integrity score higher than 7 was utilized for library preparation at the Vanderbilt Technologies for 
Advance Genomics (VANTAGE) core. Specifically, mRNA enrichment was achieved using the Ribo-Zero Kit

(Illumina) and libraries were constructed using the Illumina Tru-seq stranded mRNA sample prep kit. Sequencing

\section{chIP-on-chip}

To determine promoters bound by QseB, strain UTI89 qseB was used, that harbors a construct expressing MycHis-tagged QseB under an arabinose-inducible promoter $(12,15,27)$. As a control for non-specific pull-downs, an isogenic strain harboring the $\mathrm{pBAD}-\mathrm{MycHis} \mathrm{A}$ empty vector was used. Cultures were grown in Lysogeny Broth in the presence of $0.02 \mathrm{M}$ arabinose to ensure constant expression of QseB, at concentrations similar to those we previously published as sufficient for QseBC complementation (15). Formaldehyde was added to $1 \%$ final concentration, following the methodology as described by Mooney et al., (50). Upon addition of formaldehyde, shaking was continued for $5 \mathrm{~min}$ before quenching with glycine. Cells were harvested, washed with PBS, and stored at $-80{ }^{\circ} \mathrm{C}$ prior to analyses. Cells were sonicated and digested with nuclease and RNase A before immunoprecipitation. Immunoprecipitation was performed using an anti-Myc antibody (ThermoFisher) on six separate reactions, three for the experimental and three for the control strain. The ChIP DNA sample was amplified by ligation-mediated PCR to yield $>4 \mu \mathrm{g}$ of DNA, pooled with two other independent samples, labeled with $\mathrm{Cy} 3$ and $\mathrm{Cy} 5$ fluorescent dyes (one for the ChIP sample and one for a control input sample) and hybridized to UTI89-specific Affymetrix chips (26).

Antibiotic Survival Assays - To assess susceptibility of strains to polymyxins and aminoglycosides, strains were grown in N-minimal media in the absence (unstimulated) and presence (stimulated) of ferric iron (at a final concentration of $100 \mu \mathrm{M}$ ) as described for the transcriptional surge experiments and in Figure 2. When bacteria reached an $\mathrm{OD}_{600}$ of 0.5 , they were normalized to an $\mathrm{OD}_{600}$ of 0.5 in $5 \mathrm{ml}$ of $1 \mathrm{X}$ phosphate buffered saline (PBS) 
50

and split into two groups: A) Nothing added B) antibiotic added at a final concentration of $2.5 \mu \mathrm{g} / \mathrm{mL}$ for

polymyxin B (5X MIC), $3.125 \mu \mathrm{g} / \mathrm{mL}$ for gentamicin (1X MIC), and $10 \mu \mathrm{g} / \mathrm{mL}$ for amikacin (5X MIC). The stimulated samples also received ferric iron at a final concentration $100 \mu \mathrm{M}$. Samples were incubated for 60 or 180 minutes at $37{ }^{\circ} \mathrm{C}$ during which samples at $0,15,60$ and 180 minutes post antibiotic addition or at only 60 minutes post antibiotic addition were serially diluted and plated on nutrient agar plates (Lysogeny Broth agar) to determine colony forming units per milliliter $(\mathrm{CFU} / \mathrm{mL})$. Percent survival as a function of ferric iron prestimulation for samples incubated for one time point, 60 minutes was determined by dividing the treated samples by CFUs of the unstimulated control sample and multiplying by 100 . Percent survival as a function of ferric iron pre-stimulation for samples incubated for 180 minutes in which multiple samples at $0,15,60$ and 180 minutes was determined by dividing the treated flasks by CFUs of the $\mathrm{T}=0$ sample of each condition and multiplying by 100. For polymyxin B (PMB) survival assays performed concurrently with metabolite measurements (see relevant section below), samples were taken across time (at induction $(\mathrm{t}=0), 15,60$, and 180 minutes post ferric iron additions). For PMB survival assays performed concurrently with oxoglutarate rescue assays, oxoglutarate (5 $\mathrm{mM}$ ) was added at the same time as polymyxin B or gentamicin after standardization of the samples to an $\mathrm{OD}_{600}$ $=0.5$

\section{Metabolite Measurements}

Pellets of approximately $10^{8}$ cells were collected from PMB survival assays at each time-point. Glutamate, aspartate and coenzyme A levels were quantified using a colorimetric assay utilized from Glutamate-, Aspartateand Coenzyme A Assay Kits (all kits obtained from Sigma Aldrich) utilizing the entire, undiluted sample (10 ${ }^{8}$ cells). Assays were performed according to manufacturer's instructions in at least 2 biological replicates per strain, per timepoint.

\section{Minimum Inhibitory Concentration (MIC) Determination}

To determine the minimum inhibitory concentration of PMB, amikacin, and gentamcin in strains used in this study the broth microdilution method was used. Strains were grown at $37^{\circ} \mathrm{C}$ overnight with shaking, in cation- 
adjusted Mueller Hinton broth following clinical microbiology laboratory standard operating procedures $(51)$.

Specifically, strains were sub-cultured at a starting $\mathrm{OD}_{600}$ of 0.5 and allowed to reach growth at an $\mathrm{OD}_{600}=0.4-$ 0.5. Cells were then normalized to and $\mathrm{OD}_{600}=0.5$ or roughly $10^{5}$ cells. At this time, a 96 well polypropylene plate (for PMB) or a polystyrene plate (amikacin and gentamicin) was prepared with a gradient of concentrations (2-fold dilution) across the rows, plus a column with no antibiadded as a growth control, and a media only column to serve as a negative control. Five microliters of the standardized culture were added to each well except those holding the media control. Plates were incubated statically at $37^{\circ} \mathrm{C}$ for 24 hours. At this time, the minimum inhibitory concertation was determined. The concentration of antibiotic of the well in which bacterial growth was diminished by greater than $90 \%$ was determined to be the minimum inhibitory concentration if all three technical replicates were in agreement. Each strain was tested with 3 technical replicates and 3 biological replicates.

\section{Isolation and whole-genome sequencing of heteroresistant subpopulations from clinical isolates}

E. coli was isolated from patient urine samples and banked by MicroVU in 25\% glycerol freezer stocks. Strains were grown at $37^{\circ} \mathrm{C}$ overnight with shaking in lysogeny broth (Fisher). Strains were then diluted to $\mathrm{OD}_{600}=0.5$ in PBS, and adjusted to a $0.5 \mathrm{McF}$ arland standard. Samples were then streaked out on prepared Mueller Hinton agar plates (BD) to create a lawn, using a cotton applicator (Puritan). A single polymyxin E-test strip (Biomerieux) was placed onto the lawn. Plate were incubated at $37^{\circ} \mathrm{C}$ for 16 hours. From samples in which two populations were phenotypically present on the plate after incubation: VUTI149, 163, 184 and 247, each population (sensitive and resistant) was isolated and grown overnight at $37^{\circ} \mathrm{C}$ in lysogeny broth. The samples were then prepared for genomic DNA isolation (Qiagen). Isolated genomic DNA from both populations were sequenced (GeneWiz). Briefly, libraries were prepared using the NEBNext UltraT DNA Library Prep Kit for Illumina. The genomic DNA was fragmented by acoustic shearing with a Covaris LE220 instrument. Fragmented DNA was end repaired and adenylated. Adapters were ligated after adenylation of the 3' ends followed by enrichment by limited cycle PCR. DNA library was validated using a D100 ScreenTape on the Agilent 4200 TapeStation and was quantified using Qubit 2.0 Fluorometer. The DNA library was also quantified by real time PCR, clustered on a flow-cell and loaded on the Illumina Miseq instrument. Resulting reads were assembled using the Genome Assembly tool using 
12 SPAdes hosted on the Patric Databse. Genomes were then annotated using the Escherichia group as reference

13 using the Annotation tool hosted by Patric. To determine variation between both subpopulations (sensitive and

14 resistant), the reads of the resistant subpopulation were compared to the assembled genome of the sensitive

15 population using the Variation Analysis tool hosted on Patric's database.

\section{In vitro evolution of clinical isolate with polymyxin}

18 For clinical isolates VUTI 163 and 247 an in vitro evolution experiment was performed. Strains were grown 19 overnight at $37^{\circ} \mathrm{C}$ shaking. The next day, strains were sub-cultured 1:1000 in fresh Mueller Hinton broth with the 20 addition of a sublethal amount of polymyxin, at $0.75 \mathrm{X}$ the MIC. Samples were sub-cultured 1:1000 in fresh Muller 21 Hinton broth and polymyxin every 24 hours for 5 total passages. Genomic DNA from the second and fifth passage of VUTI63 and 247 were isolated (Qiagen) and sequenced (GeneWiz). Briefly, libraries were prepared using the NEBNext UltraT DNA Library Prep Kit for Illumina. The genomic DNA was fragmented by acoustic shearing with a Covaris LE220 instrument. Fragmented DNA was end repaired and adenylated. Adapters were ligated after adenylation of the 3' ends followed by enrichment by limited cycle PCR. DNA library was validated using a

D100 ScreenTape on the Agilent 4200 TapeStatio and was quantified using Qubit 2.0 Fluorometer. The DNA library was also quantified by real time PCR, clustered on a flowcell and loaded on the Illumina Miseq instrument. Resulting reads were assembled using the Genome Assembly tool using SPAdes hosted on the Patric Databse. Genomes were then annotated using the Escherichia group as reference using the Annotation tool hosted by

Patric. To determine variation between the passages and parental strain, the reads of the resistant subpopulation were compared to the assembled genome of the sensitive parental population using the Variation Analysis tool 32 hosted on Patric's database.

\section{Quantification and Statistical Analysis}

35 For polymyxin B survival assays, the percent survival of strains in specific conditions were calculated (mean \pm 
was displayed. For metabolite measurements, no statistical test was used, a representative of three biological

replicates was displayed. For polymyxin B survival assays across time, no statistical test was used. For analysis

10 of minimum inhibitory concentrations across passages in clinical isolates no statistical test was used, but the

H1 mean \pm SEM was displayed.

H3 Data and Code Availability

14 For whole genomes sequenced during this experiment, the sequencing data can be found on the Sequence Read 15 Archive at PRJNA625502. RNA sequencing data submission can be found on ArrayExpress at E-MTAB-9277.

t6 ChIP-on-chip data can be found in supplementary file S2 and is pending submission at ArrayExpress.

\section{Author Contributions}

$19 \mathrm{MNH}, \mathrm{CJB}$ and $\mathrm{MH}$ designed, executed and interpreted experiments, prepared figures and wrote the

;0 manuscript. TS performed statistical analyses of RNAseq output and edited the manuscript. KRG designed and

¡1 performed experiments and edited the manuscript. KAF created the $k g u R S$ deletion mutant. TB, AH, RM, SAR

;2 and DW performed and analyzed experiments and edited the manuscript.

i3 Declaration of Interests

;4 The authors declare no conflicts of interest.

\section{References}

1. M. E. Rupp, P. D. Fey, Extended spectrum beta-lactamase (ESBL)-producing Enterobacteriaceae: considerations for diagnosis, prevention and drug treatment. Drugs 63, 353-365 (2003).

2. D. L. Paterson, Recommendation for treatment of severe infections caused by Enterobacteriaceae producing extended-spectrum beta-lactamases (ESBLs). Clin Microbiol Infect 6, 460-463 (2000).

3. J. R. Johnson et al., Comparison of Escherichia coli ST131 pulsotypes, by epidemiologic traits, 19672009. Emerg Infect Dis 18, 598-607 (2012).

4. L. A. W. de Jong et al., Consecutive antibiotic use in the outpatient setting: an extensive, longitudinal descriptive analysis of antibiotic dispensing data in the Netherlands. BMC Infect Dis 19, 84 (2019).

5. S. Karve et al., The impact of initial antibiotic treatment failure: Real-world insights in patients with complicated urinary tract infection. $J$ Infect 76, 121-131 (2018). 
bioRxiv preprint doi: https://doi.org/10.1101/2020.10.22.351270; this version posted October $23,2020$. The copyright holder for this preprint (which was not certified by peer review) is the author/funder, who has granted bioRxiv a license to display the preprint in perpetuity. It is made available under aCC-BY-NC-ND 4.0 International license.

58 6. V. I. Band, D. S. Weiss, Heteroresistance: A cause of unexplained antibiotic treatment failure? PLoS

7. H. M. Zowawi et al., The emerging threat of multidrug-resistant Gram-negative bacteria in urology. Nat Rev Urol 12, 570-584 (2015).

8. M. Polat, S. S. Kara, Once-daily intramuscular amikacin for outpatient treatment of lower urinary tract infections caused by extended-spectrum $\beta$-lactamase-producing. Infect Drug Resist 10, 393-399 (2017).

9. B. W. Simpson, M. S. Trent, Pushing the envelope: LPS modifications and their consequences. Nat Rev Microbiol 17, 403-416 (2019).

10. J. C. Henderson, J. P. O'Brien, J. S. Brodbelt, M. S. Trent, Isolation and chemical characterization of lipid A from gram-negative bacteria. J Vis Exp, e50623 (2013).

11. A. Emiola, S. S. Andrews, C. Heller, J. George, Crosstalk between the lipopolysaccharide and phospholipid pathways during outer membrane biogenesis in Escherichia coli. Proc Natl Acad Sci US A 113, 3108-3113 (2016).

12. K. R. Guckes et al., Signaling by two-component system noncognate partners promotes intrinsic tolerance to polymyxin B in uropathogenic Escherichia coli. Sci Signal 10, (2017).

13. B. D. Needham, M. S. Trent, Fortifying the barrier: the impact of lipid A remodelling on bacterial pathogenesis. Nat Rev Microbiol 11, 467-481 (2013).

14. H. Lee, F. F. Hsu, J. Turk, E. A. Groisman, The PmrA-regulated pmrC gene mediates phosphoethanolamine modification of lipid A and polymyxin resistance in Salmonella enterica. $J$ Bacteriol 186, 4124-4133 (2004).

15. M. Kostakioti, M. Hadjifrangiskou, J. S. Pinkner, S. J. Hultgren, QseC-mediated dephosphorylation of QseB is required for expression of genes associated with virulence in uropathogenic Escherichia coli. Mol Microbiol 73, 1020-1031 (2009).

16. K. R. Guckes et al., Strong cross-system interactions drive the activation of the QseB response regulator in the absence of its cognate sensor. Proc Natl Acad Sci U S A, (2013).

17. J. S. Gunn et al., PmrA-PmrB-regulated genes necessary for 4-aminoarabinose lipid A modification and polymyxin resistance. Mol Microbiol 27, 1171-1182 (1998).

18. J. S. Gunn, S. S. Ryan, J. C. Van Velkinburgh, R. K. Ernst, S. I. Miller, Genetic and functional analysis of a PmrA-PmrB-regulated locus necessary for lipopolysaccharide modification, antimicrobial peptide resistance, and oral virulence of Salmonella enterica serovar typhimurium. Infect Immun 68, 6139-6146 (2000).

19. N. Kieffer et al., , an Inducible Gene Encoding an Acquired Phosphoethanolamine Transferase in Escherichia coli, and Its Origin. Antimicrob Agents Chemother 63, (2019).

20. E. J. Breland, E. W. Zhang, T. Bermudez, C. R. Martinez, M. Hadjifrangiskou, The histidine residue of QseC is required for canonical signaling between QseB and PmrB in uropathogenic Escherichia coli. $J$ Bacteriol, (2017).

21. R. Tamayo, S. S. Ryan, A. J. McCoy, J. S. Gunn, Identification and genetic characterization of PmrAregulated genes and genes involved in polymyxin B resistance in Salmonella enterica serovar typhimurium. Infect Immun 70, 6770-6778 (2002).

22. M. M. Wosten, L. F. Kox, S. Chamnongpol, F. C. Soncini, E. A. Groisman, A signal transduction system that responds to extracellular iron. Cell 103, 113-125 (2000).

23. E. A. Groisman, J. Kayser, F. C. Soncini, Regulation of polymyxin resistance and adaptation to lowMg2+ environments. J Bacteriol 179, 7040-7045 (1997).

24. M. B. Clarke, V. Sperandio, Transcriptional autoregulation by quorum sensing Escherichia coli regulators B and C (QseBC) in enterohaemorrhagic E. coli (EHEC). Mol Microbiol 58, 441-455 (2005).

25. E. J. Breland, A. R. Eberly, M. Hadjifrangiskou, An Overview of Two-Component Signal Transduction Systems Implicated in Extra-Intestinal Pathogenic E. coli Infections. Front Cell Infect Microbiol 7, 162 (2017).

26. M. Hadjifrangiskou et al., A central metabolic circuit controlled by QseC in pathogenic Escherichia coli. Mol Microbiol 80, 1516-1529 (2011).

27. K. R. Guckes et al., Strong cross-system interactions drive the activation of the QseB response regulator in the absence of its cognate sensor. Proc Natl Acad Sci U S A 110, 16592-16597 (2013). 
28. D. Shin, E. J. Lee, H. Huang, E. A. Groisman, A positive feedback loop promotes transcription surge that jump-starts Salmonella virulence circuit. Science 314, 1607-1609 (2006).

29. A. Kato, H. D. Chen, T. Latifi, E. A. Groisman, Reciprocal control between a bacterium's regulatory system and the modification status of its lipopolysaccharide. Mol Cell 47, 897-908 (2012).

30. R. McClure et al., Computational analysis of bacterial RNA-Seq data. Nucleic Acids Res 41, e140 (2013).

31. A. R. Wattam et al., PATRIC, the bacterial bioinformatics database and analysis resource. Nucleic Acids Res 42, D581-591 (2014).

32. J. D. Rabinowitz, E. Kimball, Acidic acetonitrile for cellular metabolome extraction from Escherichia coli. Anal Chem 79, 6167-6173 (2007).

33. W. Cai et al., A novel two-component signaling system facilitates uropathogenic Escherichia coli's ability to exploit abundant host metabolites. PLoS Pathog 9, e1003428 (2013).

34. J. R. Johnson et al., Molecular epidemiological analysis of Escherichia coli sequence type ST131 $(\mathrm{O} 25: \mathrm{H} 4)$ and blaCTX-M-15 among extended-spectrum-beta-lactamase-producing E. coli from the United States, 2000 to 2009. Antimicrob Agents Chemother 56, 2364-2370 (2012).

35. J. R. Brannon et al., Invasion of vaginal epithelial cells by uropathogenic Escherichia coli. Nat Commun 11, 2803 (2020).

36. R. S. Hanson, D. P. Cox, Effect of different nutritional conditions on the synthesis of tricarboxylic acid cycle enzymes. J Bacteriol 93, 1777-1787 (1967).

37. M. Marcus, Y. S. Halpern, The metabolic pathway of glutamate in Escherichia coli K-12. Biochim Biophys Acta 177, 314-320 (1969).

38. T. J. Goss, A. Perez-Matos, R. A. Bender, Roles of glutamate synthase, gltBD, and gltF in nitrogen metabolism of Escherichia coli and Klebsiella aerogenes. J Bacteriol 183, 6607-6619 (2001).

39. A. Reitzer, Biosynthesis of glutamate, aspartate, asparagine, L-alanine, and D-alanine. Escherichia coli and Salmonella: Cellular and molecular biology. Chapter 3.6.1.3, (2004).

40. R. Kumar, K. Shimizu, Metabolic regulation of Escherichia coli and its gdhA, glnL, gltB, D mutants under different carbon and nitrogen limitations in the continuous culture. Microb Cell Fact 9, 8 (2010).

41. D. De Biase, A. Tramonti, F. Bossa, P. Visca, The response to stationary-phase stress conditions in Escherichia coli: role and regulation of the glutamic acid decarboxylase system. Mol Microbiol 32, 1198-1211 (1999).

42. K. M. Belaynehe et al., Emergence of mcr-1 and mcr-3 variants coding for plasmid-mediated colistin resistance in Escherichia coli isolates from food- producing animals in South Korea. Int J Infect Dis $\mathbf{7 2}$, 22-24 (2018).

43. Y. Y. Liu et al., Emergence of plasmid-mediated colistin resistance mechanism MCR-1 in animals and human beings in China: a microbiological and molecular biological study. Lancet Infect Dis 16, 161-168 (2016).

44. J. Koch-Weser et al., Adverse effects of sodium colistimethate. Manifestations and specific reaction rates during 317 courses of therapy. Ann Intern Med 72, 857-868 (1970).

45. S. P. Conway et al., Intravenous colistin sulphomethate in acute respiratory exacerbations in adult patients with cystic fibrosis. Thorax 52, 987-993 (1997).

46. M. J. Ledson, M. J. Gallagher, C. Cowperthwaite, R. P. Convery, M. J. Walshaw, Four years' experience of intravenous colomycin in an adult cystic fibrosis unit. Eur Respir J 12, 592-594 (1998).

47. M. E. Falagas, S. K. Kasiakou, Colistin: the revival of polymyxins for the management of multidrugresistant gram-negative bacterial infections. Clin Infect Dis 40, 1333-1341 (2005).

48. S. P. Conway et al., Safety and tolerability of bolus intravenous colistin in acute respiratory exacerbations in adults with cystic fibrosis. Ann Pharmacother 34, 1238-1242 (2000).

49. L. S. Gonzalez, J. P. Spencer, Aminoglycosides: a practical review. Am Fam Physician 58, 1811-1820 (1998).

50. R. A. Mooney et al., Regulator trafficking on bacterial transcription units in vivo. Mol Cell 33, 97-108 (2009). 
bioRxiv preprint doi: https://doi.org/10.1101/2020.10.22.351270; this version posted October 23, 2020. The copyright holder for this preprint (which was not certified by peer review) is the author/funder, who has granted bioRxiv a license to display the preprint in perpetuity. It is made available under aCC-BY-NC-ND 4.0 International license.

70

71

72

73

74

75

76

77

78

79

30

A

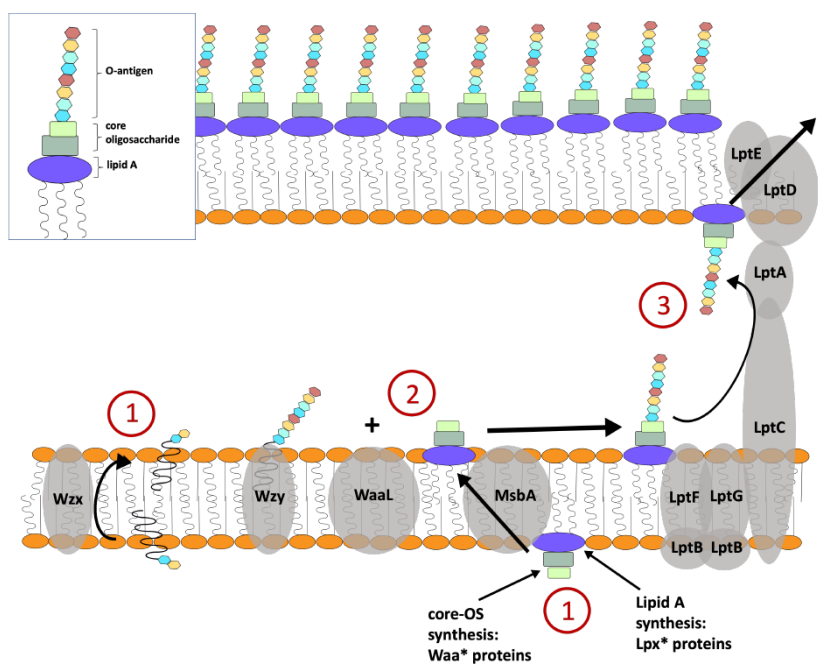

C

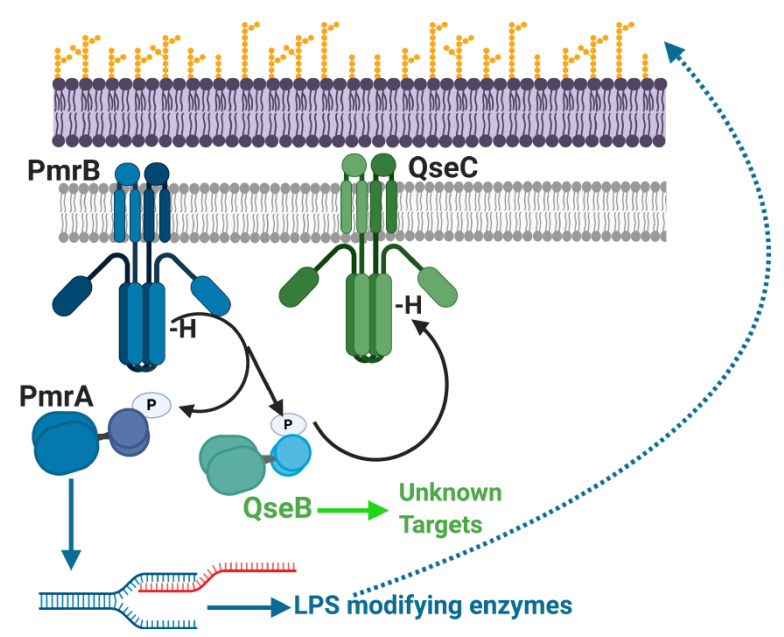

B

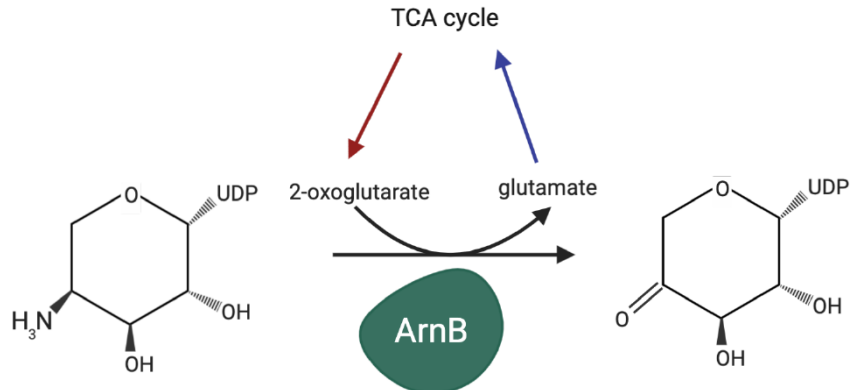

UDP-2-keto-pyranose
UDP-B-L-Ara4N

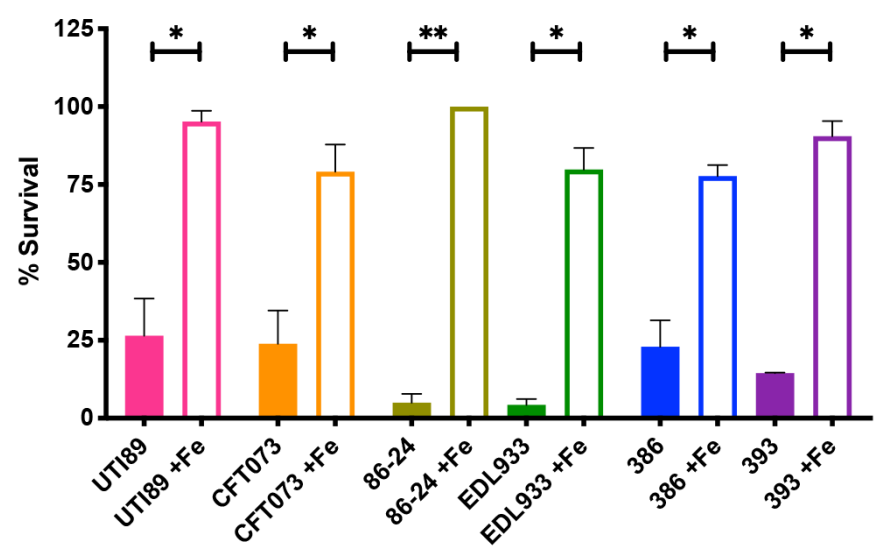


Figure 1: QseBC-PmrAB mediated control of polymyxin B resistance in $\boldsymbol{E}$. coli. ( (A) Cartoon depicts - in a simplified manner - the steps in lipopolysaccharide (LPS) biosynthesis in Escherichia coli. (B) Schematic shows the ArnB-mediated reaction that converts UDP-2-ketopyranose to UDP-b-L-Ara4N by consuming oxoglutarate and producing glutamate. (C) Cartoon depicts the mechanism of activation of the two-component systems PmrAB (blue) and QseC (green). PmrB is a membrane bound histidine kinase that is activated by ferric iron. Upon activation it auto-phosphorylates and then transfers the phosphoryl group onto its cognate response regulator PmrA and the non-cognate QseB. PmrA is a transcription factor, regulating the transcription of LPS modifying genes. QseB is also transcription factor, whose targets are unknown. (D) Graph depicts results of polymyxin B survival assays for each strain. Cells were allowed to reach mid logarithmic growth phase in the presence or absence of ferric iron and normalized. Cells were then exposed to polymyxin B at $2.5 \mu \mathrm{g} / \mathrm{mL}$ or to diluent alone (sterile water), for one hour. At this time cells were serially diluted and plated to determine colony forming units per $\mathrm{mL}$. To determine percent survival, cells exposed to polymyxin were compared to the untreated controls (mean $\pm \mathrm{SEM}, \mathrm{n}=3$ ). To determine statistical significance, a Welch's T-test was performed between the untreated strain and the same strain treated with ferric iron. $(*), p<0.05 ;(* *), p<0.01$.
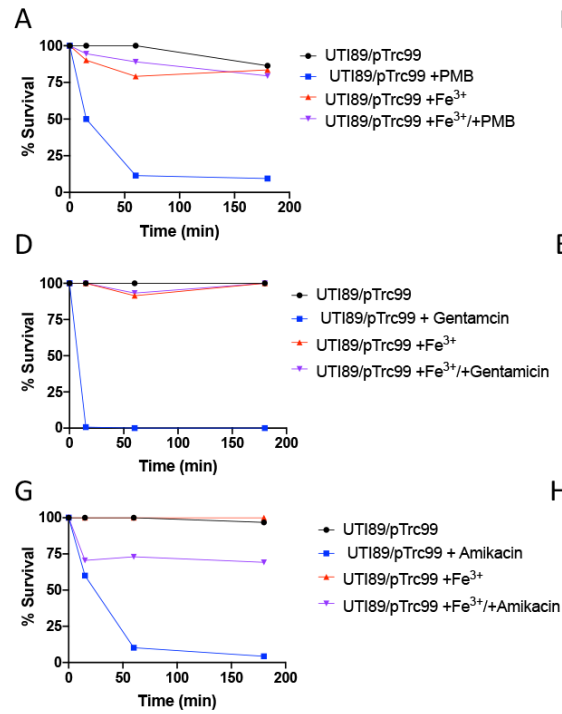

- UT189/PTrc99 - UTI89/pTrc99 + Amikacin $\star U T 189 / \mathrm{PTC}^{2} 99+\mathrm{Fe}^{3+}$ * UTI89/PTrc99 +Fe $e^{3+} /+$ Amikacin

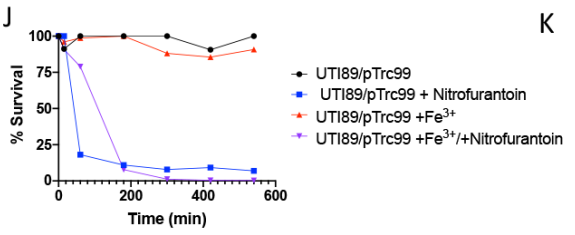

B

E

$\mathrm{H}$
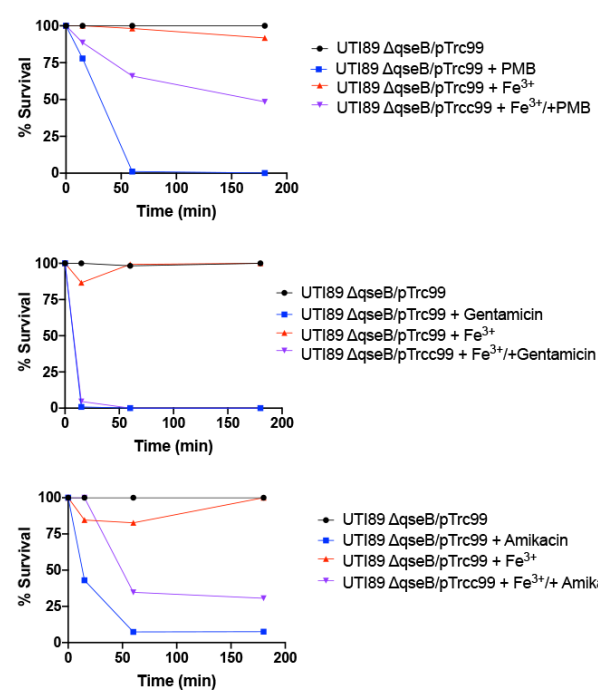

- UT189 $\Delta$ qseB/pTrc99

- UT189 $\Delta q s \in B / p T r c 99+$ Amikaci

- UT189 $\Delta$ qseB/pTrc99 + $\mathrm{Fe}^{3+}$
- UT189 $\Delta \mathrm{qseB} / \mathrm{pTrcc} 99+\mathrm{Fe}^{3+}+$ +Amikacin

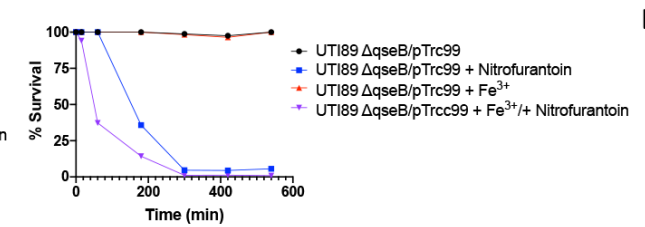

$\mathrm{C}$

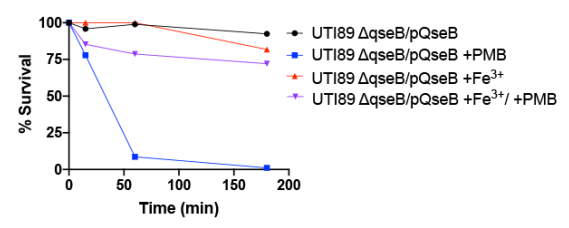

$\mathrm{F}$
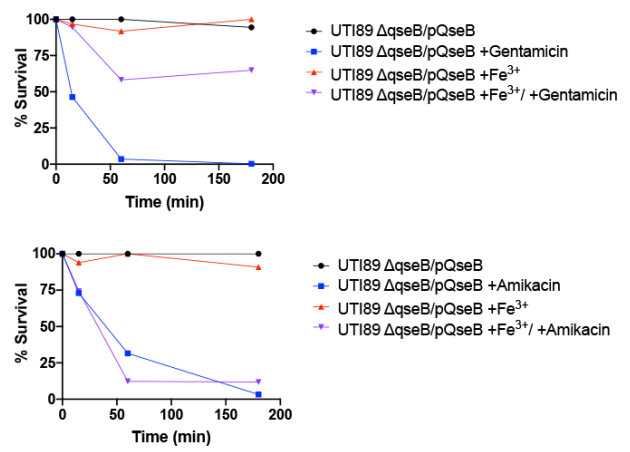
UTisg $\triangle \mathrm{qseB} / \mathrm{pQseB}+$ Amikacin $-189 \Delta q s e B / p Q s e B+\mathrm{Fe}^{3+}$ UTI89 $\Delta q$ seB/pQseB $+\mathrm{Fe}^{3+} /+$ Amikacin

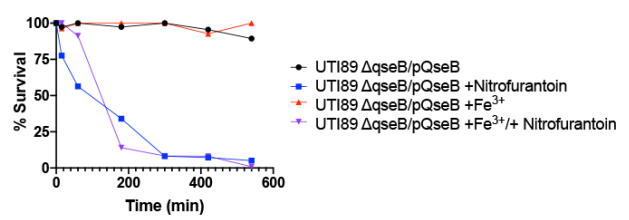

Figure 2: QseB confers resistance to positively charged antibiotics. Graphs depict survival across time during antibiotic challenge of isogenic strains lacking or harboring QseB. In each experiment, strains were challenged with: no additives (Black lines, control); $100 \mu \mathrm{M}$ ferric iron (Red lines; PmrB activation stimulus); antibiotic of choice (Blue lines), or; both $100 \mu \mathrm{M}$ ferric iron and an antibiotic of choice (Purple lines). (A-C) Polymyxin survival assays with UTI89/pTrc99 (A); UTI89 4 qseB/pTrc99 (B), or UTI89 $4 q s e B / p Q s e B$. (D-F) Gentamicin survival assays with UTI89/pTrc99 (D); UTI89 $\Delta q s e B / p \operatorname{Trc} 99$ (E), or UTI89 $q q s e B / \mathrm{pQseB}$ (F). (G-I) Amikacin survival assays with UTI89/pTrc99 (G); UTI89 $q$ seB/pTrc99 (H), or UTI89 $q$ seB/pQseB (I) (J-L) Nitrofurantoin 
A

B representative of three trials $(n=3)$.

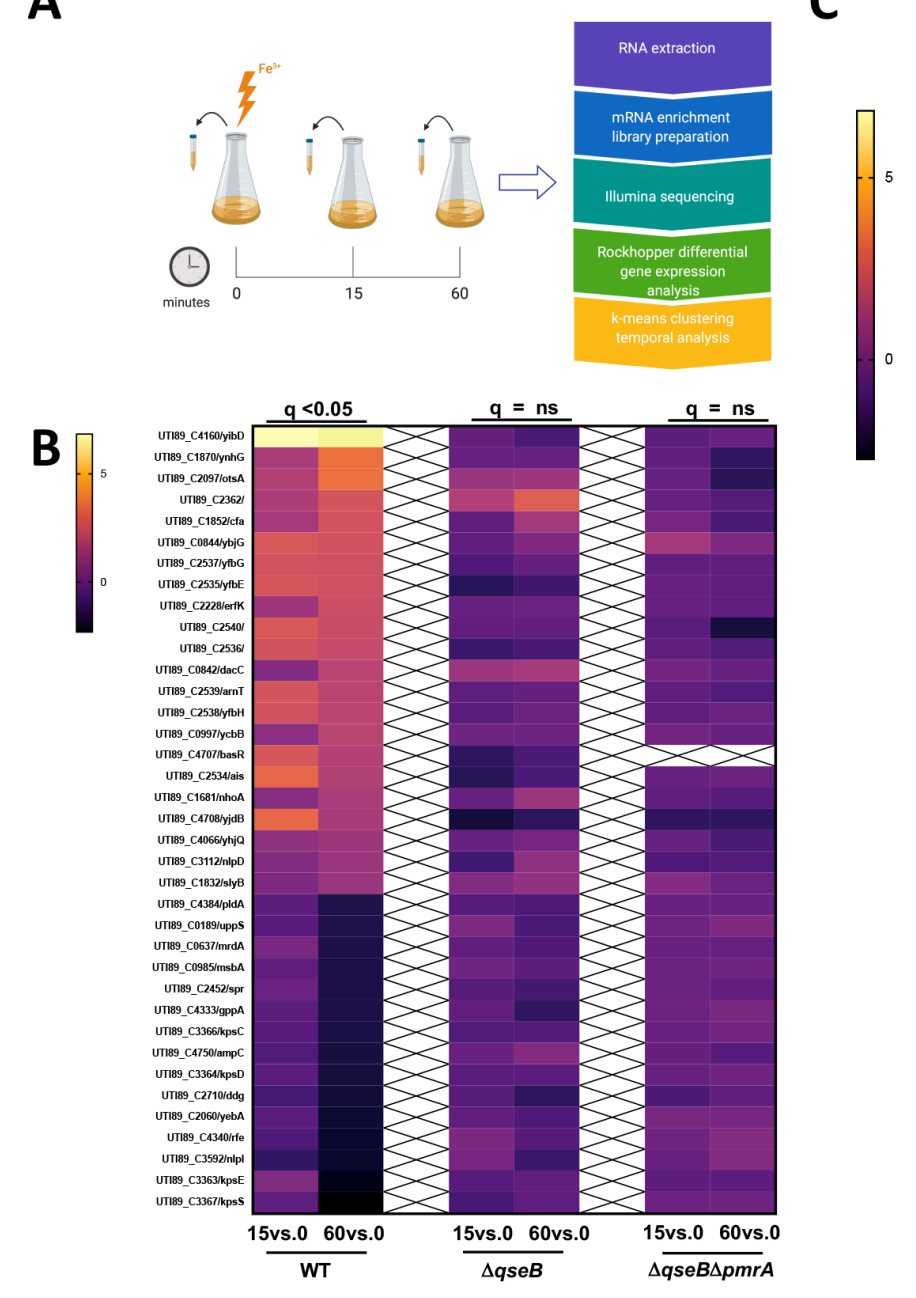

survival assays with UTI89/pTrc99 (J); UTI89 $q$ qseB/pTrc99 (K), or UTI89 $q$ seB/pQseB (L). All graphs show a

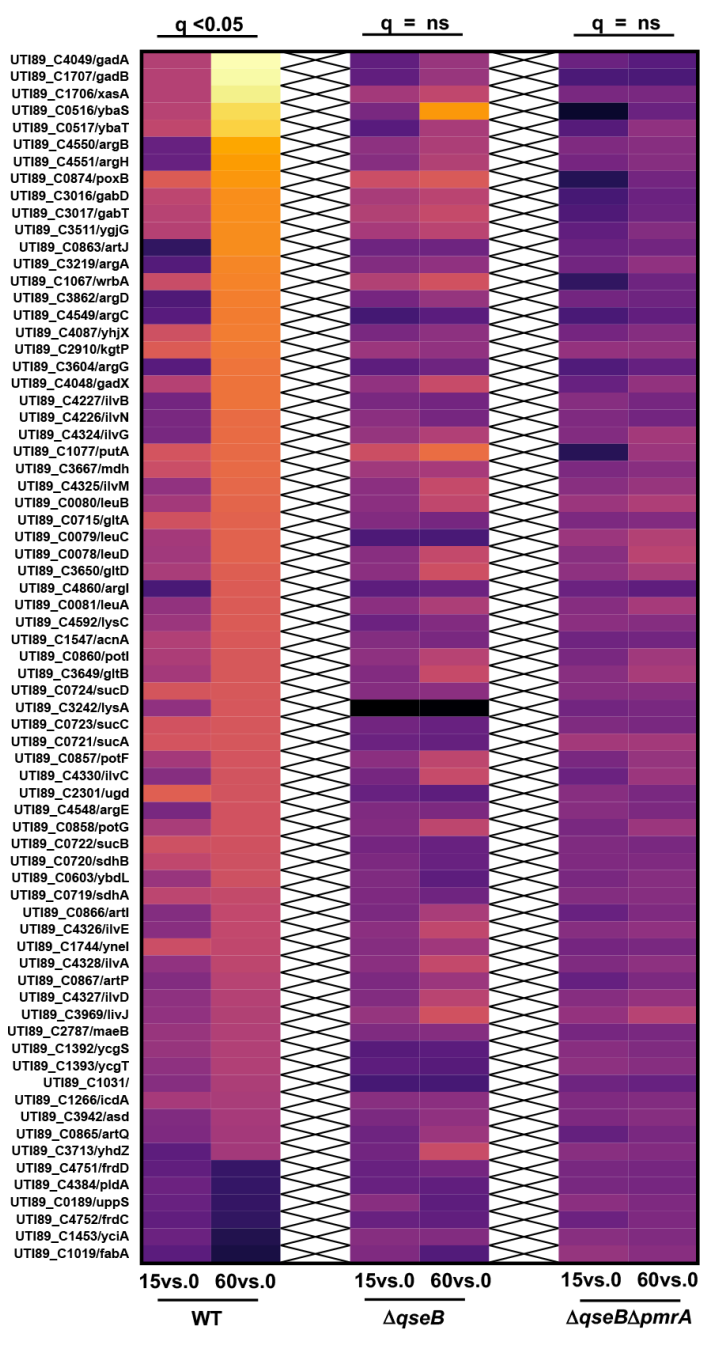

Figure 3: RNAseq profiling across time reveals a metabolic circuit controlled by QseB and PmrA. (A) Schematic showing the pipeline for sample collection and data processing. (B-C) Heatmaps indicate $\log _{2}$ relative fold change of UTI89 WT, $\Delta q s e B$, and $\triangle q s e B \Delta p m r A$ for genes involved in cell envelope modification (B) or metabolism (C) after stimulation with ferric iron at 15- and 60-minutes post stimulation. These genes were significantly $(\mathrm{q}<0.05)$ changed at 60 minutes compared to pre-stimulation $(\mathrm{T}=0)$ in wild-type UTI89. (C) A 
bioRxiv preprint doi: https://doi.org/10.1101/2020.10.22.351270; this version posted October 23, 2020. The copyright holder for this preprint (which was not certified by peer review) is the author/funder, who has granted bioRxiv a license to display the preprint in perpetuity. It is made available under aCC-BY-NC-ND 4.0 International license.

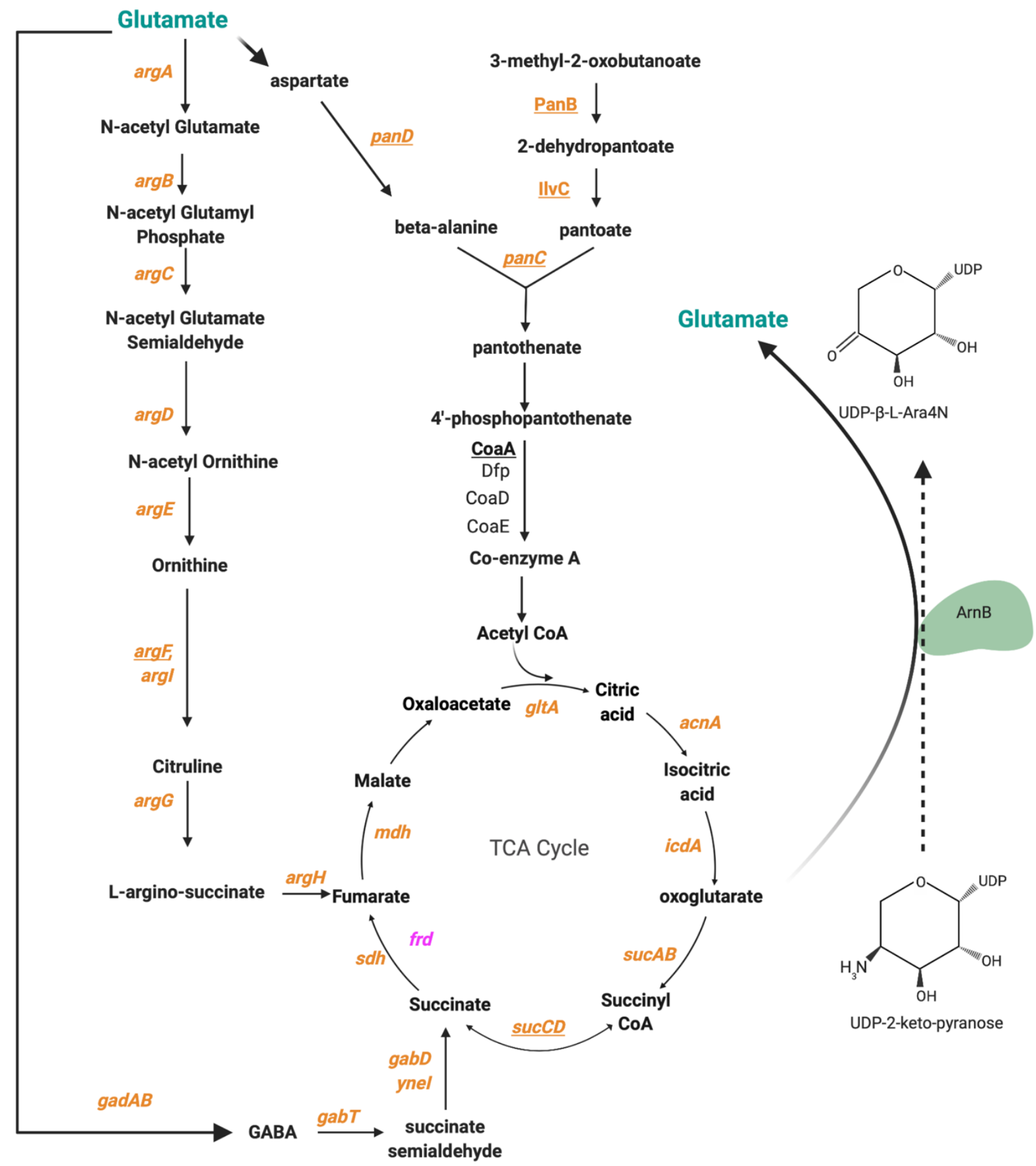

t0 heatmap showing $\log _{2}$ relative fold change of UTI89 WT, $\Delta q s e B$, and $\Delta q s e B \Delta p m r A$ for genes in metabolic pathways after stimulation with ferric iron at 15 - and 60 -minutes post stimulation. These genes were significantly $(\mathrm{q}<0.05)$ changed at 60 minutes compared to pre-stimulation $(\mathrm{T}=0)$ in wild-type UTI89, but not in the mutant 13 strains. 
A

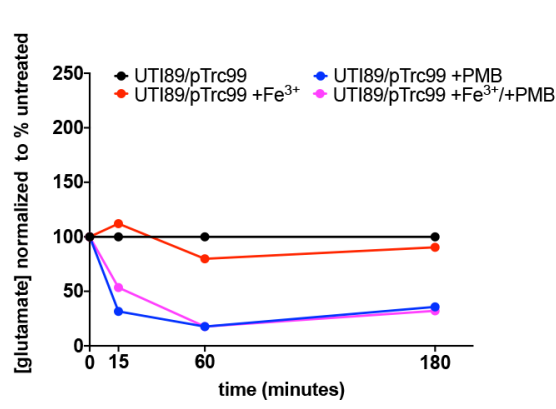

D

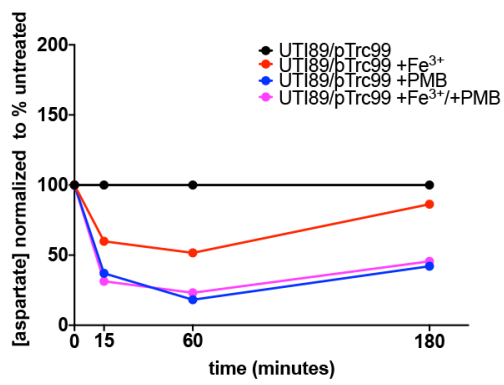

G

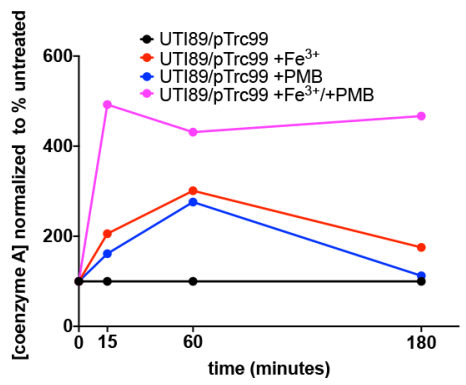

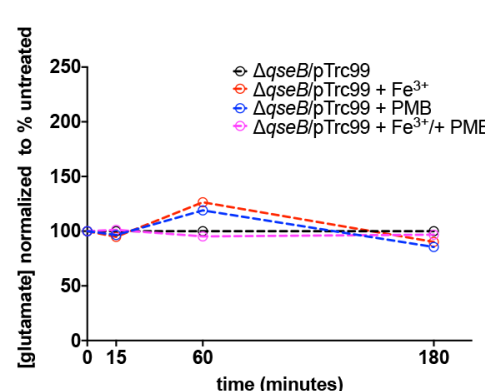

E

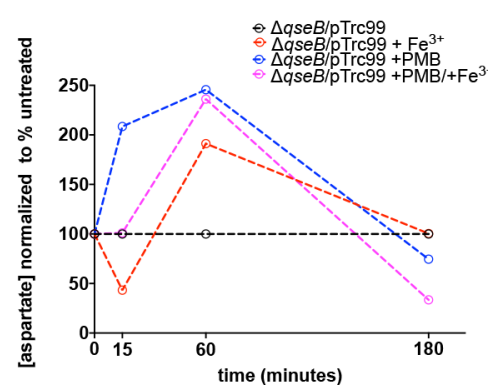

$\mathrm{H}$

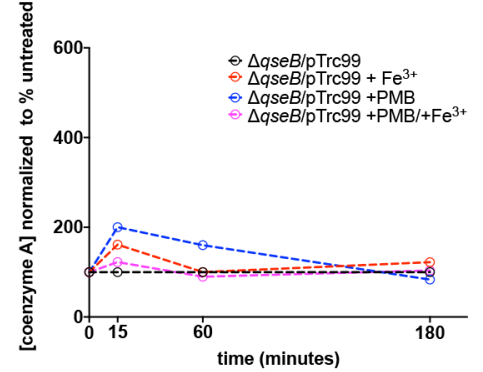

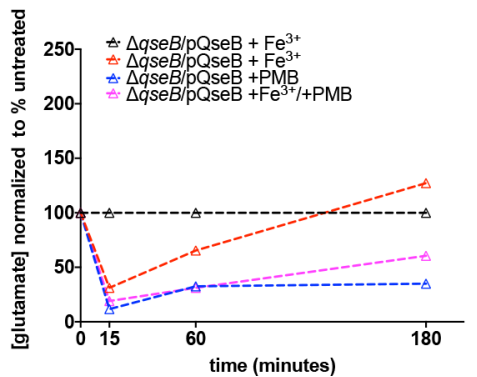

F
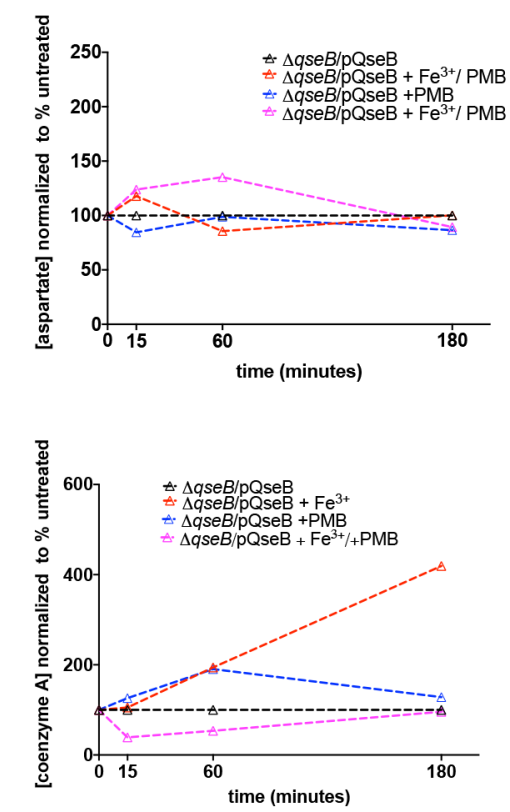

Figure 5: Metabolomic changes in response to LPS modification and QseB control. Graphs indicate metabolite abundance for glutamate, aspartate and co-enzyme A over time in wild-type pathogenic E. coli and isogenic mutants under different stimulation conditions. Measurements are normalized to a sample in which no additives or conditions were changed (black lines). Pink lines show measurements from samples in which ferric iron and polymyxin were added. Blue lines show measurements from samples in which only polymyxin was added. Red lines show measurements in which only ferric iron was added. (A-C) Glutamate was measured across time in wild-type UTI89 (A), UTI89 $q s e B / p T r c 99$ (B) and UTI89 $4 q s e B / p Q s e B$ (C). (D-F) Aspartate was measured in wild-type UTI89 (D), UTI89 $q$ seB/pTrc99 and (E) UTI89 $\Delta q s e B / p Q s e B$ (F). (G-I) Aspartate was measured in wild-type UTI89 (G), UTI89 4 seB/pTrc99 (H), and UTI89 $q$ seB/pQseB (I). Graphs are representative of at least three biological repeats. 
A

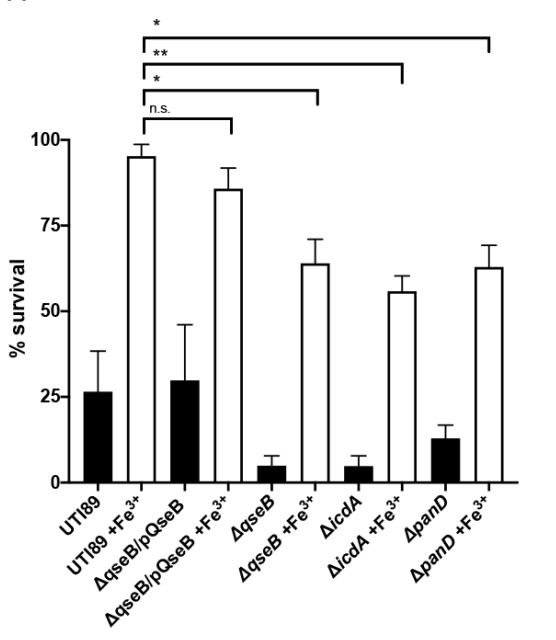

B

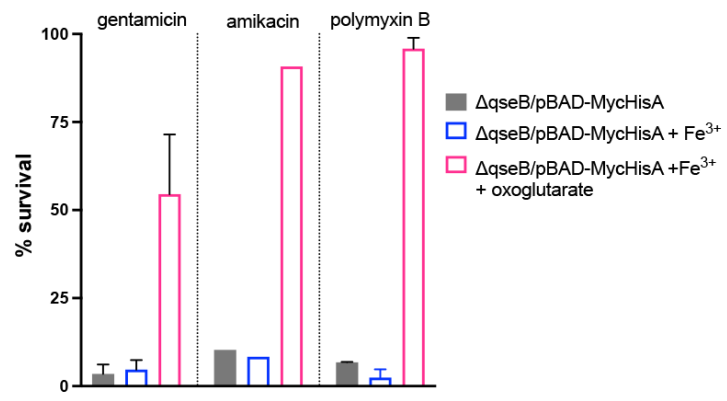

C

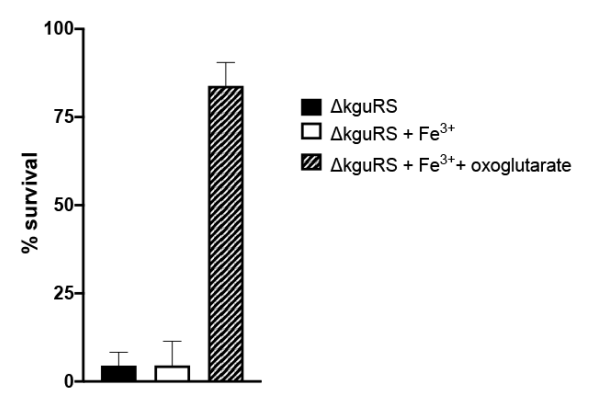

Figure 6: Oxoglutarate restores antibiotic resistance in the absence of QseB or an oxoglutarate sensor. (A) Graph depicts polymyxin B survival assays for wild-type $E$. coli and isogenic mutants deleted for $q s e B$, icd $A$, or panD. Cells were allowed to reach mid logarithmic growth phase in the presence or absence of ferric iron and normalized. Cells were then either exposed to polymyxin at $2.5 \mu \mathrm{g} / \mathrm{mL}$ or without addition for one hour. To determine percent survival, cells exposed to polymyxin were compared to those that were not (mean \pm SEM, $\mathrm{n}=$ 3). To determine statistical significance, a Welch's T-test was performed between strains treated with ferric iron and UTI89 wild-type treated with ferric iron. $(*), p<0.05 ;(* *), p<0.01 ;(* * *), p<0.001$. N.S. denotes a comparison that did not result in statistical significance. (B) Graph depicts gentamicin, amikacin and polymyxin B survival assays for $E$. coli isogenic mutants deleted for qseB and carrying the pBAD-MycHisA plasmid. Cells were allowed to reach mid logarithmic growth phase in the presence or absence of ferric iron and normalized. Cells were then exposed to gentamicin at $3.125 \mu \mathrm{g} / \mathrm{mL}$, amikacin at $10 \mu \mathrm{g} / \mathrm{mL}$ or polymyxin B at $2.5 \mu \mathrm{g} / \mathrm{mL}$ or without addition for one hour. Cells were also exposed to ferric iron $(100 \mu \mathrm{M})$ and/or oxoglutarate $(5 \mathrm{mM})$. To determine percent survival, cells exposed to antibiotic were compared to those that were not (mean \pm SEM, $n=3$ for gentamicin and polymyxin B assay, $\mathrm{n}=1$ for amikacin assay). (C) Graph depicts polymyxin B survival assay for $E$. coli isogenic mutants of $\mathrm{kguRS}$ an oxoglutarate sensing two-component system. Cells were allowed to reach mid logarithmic growth phase in the presence or absence of ferric iron and normalized. Cells were then exposed to polymyxin at $2.5 \mu \mathrm{g} / \mathrm{mL}$ or without addition for one hour. Cells were also exposed to ferric iron $(100 \mu \mathrm{M})$ and/or oxoglutarate $(5 \mathrm{mM})$. Percent survival was determined as described above and in the materials and methods. 
A

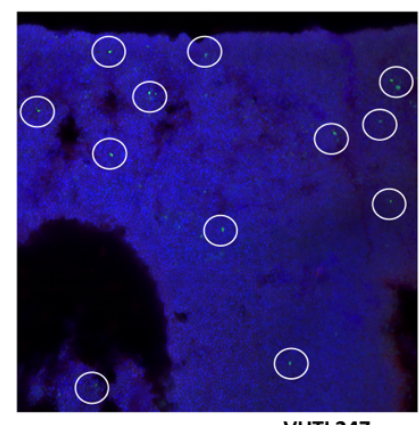

C

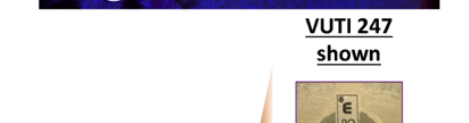

F

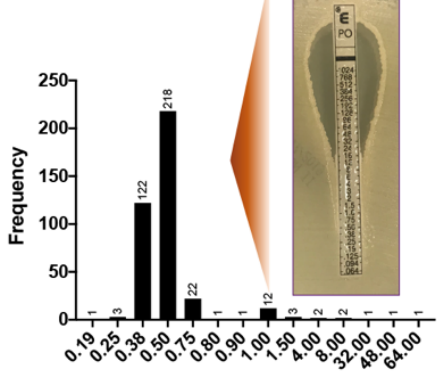

MIC

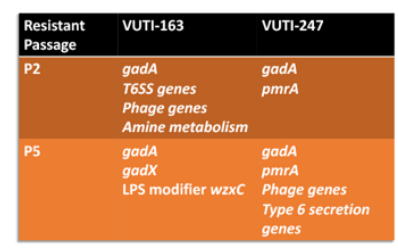

B

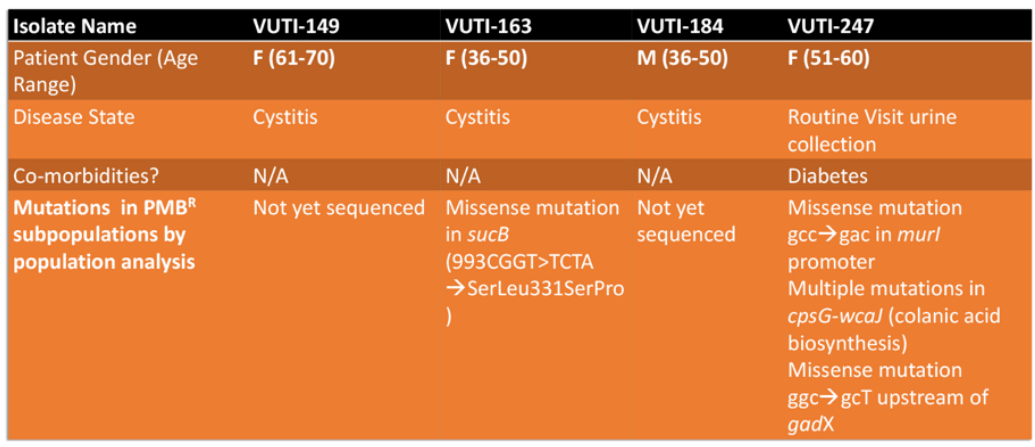

D

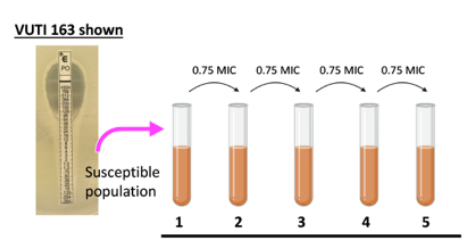

E

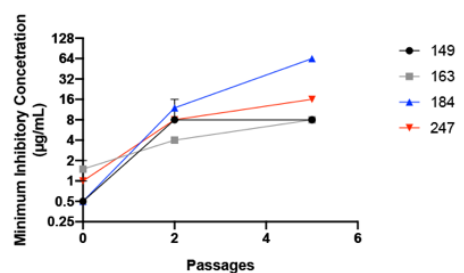

G

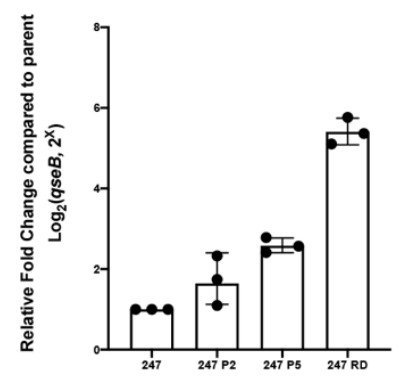

H

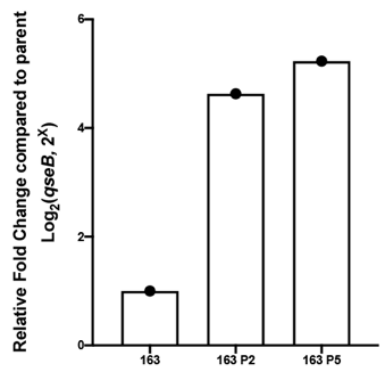

Figure 7: SNPs associated with polymyxin B-resistant subpopulations in clinical $E$. coli isolates. (A) Confocal Laser Scanning Microscopy (CLSM) images of E. coli biofilms formed by bacteria harboring the previously constructed Pqse::GFP reporter. The Pqse::GFP reporter is expressed in a small number of cells (marked with circles). Blue coloration is Topro-3 DNA stain. Images depicted are representative of at least 5 biological replicates. (B) Table depicts demographics associated with the strain source patients. Whole genome sequencing of VUTI 247 and VUTI163 reveals mutations compared to the susceptible subpopulation (see also supplementary file). (C) Graph shows the distribution of polymyxin B MICs for 300 E. coli urinary tract isolates collected at Vanderbilt University Medical Center Clinical laboratory under IRB\#151465. MICs were determined using polymyxin E-test strips. Inset image depicts VUTI 247 exhibiting two populations with differing MIC's: a major subpopulation with a susceptible MIC and a minor, yet stable, subpopulation with a resistant MIC. 2). (D) Schematic depicts the set-up for an in vitro evolution experiment. The susceptible population from each tested isolate demonstrating two populations (VUTI 149, VUTI 163, VUTI 184 and VUTI 247), was passaged 5 times, over a 5-day period, at $0.75 \mathrm{X}$ its polymyxin B MIC. (E) The MIC prior to passage, the second passage, and the fifth passage was determined using broth microdilution. Graph depicts the minimum inhibitory concentration of a strain over each passage (mean \pm SEM, $n=3$ ). (F) Table contains the mutations that were found after passages 2 and 5 of VUTI 163 and VUTI 247 underwent whole genome sequencing and were compared to the parent strains (See also supplementary file 2). (G) VUTI247, passage 2 and 5 and the resistant 
15 daughter from the resistant subpopulation were tested for activity of the qse operon. Cells were collected from a t6 culture grown overnight allowed to reach stationary phase. RNA was extracted and reverse -transcribed. cDNA 17 resulting from the reaction was subjected to $\mathrm{qPCR}$ with a probe complementary to the $q s e B$ region. Graph depicts $\log _{2}$ (fold change) of $q s e B$ transcripts at each time point relative to the parental VUTI163 (mean $\pm \mathrm{SEM}, \mathrm{n}=3$ ). $(\mathbf{F})$ 19 VUTI247 and passage 2 and 5 were tested for activity of the qse operon. Cells were collected from a culture ;0 grown overnight allowed to reach stationary phase. RNA was extracted and reverse -transcribed. cDNA resulting ;1 from the reaction was subjected to qPCR with a probe complementary to the qseB region. Graph $\operatorname{depicts} \log _{2}$ (fold change) of $q s e B$ transcripts at each time point relative to the parental VUTI163 (mean $\pm \mathrm{SEM}, \mathrm{n}=1$ ). 